\title{
Chiome a confronto Analisi semiotica delle acconciature di Donald Trump e Kim Jong-un
}

\author{
Giampaolo Proni \\ Università di Bologna, Dipartimento di Scienze per la Qualità della Vita \\ giampaolo.proni@unibo.it \\ https://www.unibo.it/sitoweb/giampaolo.proni
}

\begin{abstract}
Nel rapporto tra due leader politici quali Donald Trump e Kim Jong-un le rispettive acconciature colpiscono per la loro originalità. In questo articolo ho cercato di eseguire un'analisi semiotica delle due pettinature, considerate dei testi costruiti e enunciati come elementi della presentazione di sé in pubblico. Ho evidenziato, nel caso del leader nordcoreano, il fatto che la lettura della sua immagine comporta problemi di trans-culturalità. L'acconciatura è un elemento del vestito, secondo la definizione di Barthes e della sociologia dell'abbigliamento. Nell'analisi ho adottato i concetti della semiotica interpretativa nei seguenti passi: livello espressivo; dimensione paradigmatica; livello dell'interpretante nelle tre fasi della teoria di Peirce. Ho adottato un approccio al vestito come discorso prevalentemente fatico secondo le posizioni di Malinowski/Jakobson/Goffman. Propongo una teoria degli inganni palesi per spiegare gli accordi di reciproco riconoscimento delle mascherature quali la tinta dei capelli e il make-up in generale. L'acconciatura di Trump si spiega come l'adozione di un idioletto di presentazione di sé, mentre quella di Kim costituisce un caso di linguaggio dinastico. La sua interpretazione risulta molto diversa in Corea del Nord e in Occidente. I capelli di Trump evidenziano una sorta di 'autismo nella presentazione di sé', mentre nel caso di Kim questa separazione è estesa alla sua cultura nel complesso. In conclusione, le conseguenze congiunte di queste caratteristiche dei due personaggi portano a ipotizzare che il dialogo che hanno intrapreso possa restare a livello di esibizione del contatto e non arrivare mai a un vero sostanziale confronto.
\end{abstract}

\section{Parole chiave}

Donald Trump, acconciatura, pettinatura, semiotica, sociosemiotica, analisi semiotica

\begin{abstract}
In the relationship between two political leaders such as Donald Trump and Kim Jongun, both the hairstyles strike attention for their originality. In this article I have tried to perform a semiotic analysis of the two hairdos, considered as texts constructed and enunciated as elements of self-presentation in public. In the case of the North Korean leader, I highlighted the fact that the reading of his image entails problems of trans-culturality. The hairstyle is an element of the dress, according to the definition of Barthes and the sociology of clothing. In my analysis I adopted the concepts of interpretive semiotics in the following steps: expressive level; paradigmatic dimension; interpretant level in the three phases of Peirce's theory. I have adopted an approach to dress as a predominantly phatic discourse according to the positions of Malinowski/ Jakobson/Goffman. I p ropose the theory of exposed deception to explain the agreements of mutual recognition of masking such as hair dye and make-up in general. Trump's hairstyle is explained as the adoption of an idiolect of self-presentation, while Kim's is a case of dynastic language. He is interpreted in very different ways in North Korea and the West. Trump's hair suggests a kind of 'autism in self-presentation', while in Kim's case the separation extends to his culture as a whole. In conclusion, the
\end{abstract}




\section{Dcula \\ Occhio semiotico sui media | Semiotic eye on media}

Giampaolo Proni | Chiome a confronto | DOI: 10.12977/ocula2019-14

combined consequences of these characteristics of the two personalities lead to the hypothesis that the dialogue they have undertaken may remain less a true substantial confrontation than a mere display of contact.

\section{Key Words}

Donald Trump, haircut, hairdo, semiotics, sociosemiotics, semiotic analysis

\section{Sommario}

1. Introduzione

2. Il campione dei testi analizzati

3. Traslazione di un testo da una cultura a un'altra

4. L'acconciatura come elemento del vestito

5. Trump e Kim: costume o abbigliamento?

6. Livello espressivo

6.1 Trump: l'enigma del riporto

6.2 Kim: dinastie comuniste e pettinature

6.3 La dimensione paradigmatica

7. Livello del contenuto: la funzione fatica del vestito

7.1 La mascheratura di Trump

7.2 Gli inganni palesi dell'immagine pubblica

7.3 Funzione e significato di Kim Jong-un

7.3.1 Il regime nord coreano e l'acconciatura maschile

7.3.2 Kim in occidente

7.3.3 Kim: la narrazione dell'uguaglianza

7.3.4 La circolazione dei valori estetici in due diversi sistemi

7.4 L'interpretazione personale delle fiction sociali: l'idioletto di Trump

7.4.1 Il significato dei linguaggi

7.4.2 Kim: un costume dinastico

7.4.3 Kim e l'estetica occidentale

7.4.4 Che cosa Kim vuole dire con la propria immagine?

8. Terzo livello: il significato pragmatico

8.1 Conseguenze ipotizzabili della pettinatura di Trump

8.2 Conseguenze ipotizzabili della pettinatura di Kim e conclusione

Riferimenti

Testi Campione

Bibliografia 


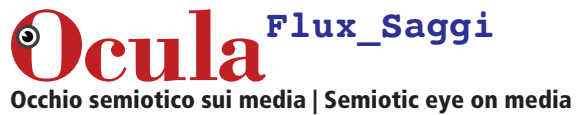

Giampaolo Proni | Chiome a confronto | DOI: 10.12977/ocula2019-14

\section{Introduzione}

Oggi due personalità famose nel mondo hanno riportato l'acconciatura maschile sotto i riflettori: Donald Trump, presidente degli USA, e Kim Jongun, il Leader Supremo della Corea del Nord.

Fin dall'inizio della campagna presidenziale del 2016 i capelli di Trump hanno attirato l'attenzione dell'opinione pubblica e dei media per bizzarria e originalità.

Le due immagini pubbliche hanno però caratteristiche diverse. Mentre il presidente degli Stati Uniti è da tempo una celebrity, Kim non si forma (semioticamente) dentro il sistema occidentale dei media, ma in relazione a quello della Corea del Nord, uno dei paesi più chiusi del mondo ${ }^{1,2}$.

Quando da questo sistema isolato viene proiettato in quello globale, Kim ovviamente cambia, così come un uomo in frac è diverso se sale sul podio di un'orchestra o se invece scende nella pista di un circo.

Si tratterà dunque, nel caso di Kim, di un'analisi transculturale.

Per i casi della storia, accade che queste due capigliature si siano scontrate e incontrate in quanto tratti di due capi di stato impegnati in un complesso confronto. Questo ha portato a un inevitabile paragone mediatico: foto contro foto, video contro video. L'analisi semiotica che segue cerca di rispondere alla domanda "Che cosa significano questi due testi contrapposti sui media?".

\section{Il campione dei testi analizzati}

Il campione di testi scelti per l'analisi è una raccolta di letture e testi verbali e visivi reperiti sul web. Ad essi si devono aggiungere le informazioni che come autore ho letto, visto e memorizzato sull'argomento. Il campione effettivo comprende un certo numero di immagini e pagine scritte che descrivo-

1 Anche i dati biografici di Kim Jong-un sono incerti: vedi Wikipedia Kim Jongun (URL in Riferimenti).

2 Modalità di citazione della fonte Wikipedia come opera enciclopedica. Ogni riferimento alla voce di Wikipedia viene usato alle seguenti condizioni: a) si tratta a giudizio dell'autore di informazione difficilmente contestabile, nella quale i margini di errore di Wikipedia si possono considerare pari a quelli di qualsiasi enciclopedia; b) viene citata la voce in inglese perché è solitamente quella (tra le lingue che l'autore legge) con maggiore estensione e bibliografia più completa. Per ogni voce sono state generalmente consultate anche le voci in italiano e francese. È possibile attraverso lo strumento di traduzione dei browser leggere, sia pure con qualche imprecisione, le traduzioni in qualsiasi lingua. Quando era disponibile è stata dunque consultata la voce in coreano nella traduzione automatica in inglese, anche se i risultati non sono del tutto chiari. Le voci in coreano di Wikipedia, tuttavia, sono probabilmente state scritte da sud coreani, ma le fonti citate (per esempio riguardo alla data di nascita di Kim Jong-Un) spesso considerano anche fonti ufficiali nord coreane. c) Si è evitato in ogni caso di citare o fare riferimento a giudizi politici, etici o ideologici relativi alla Corea del Nord. 


\section{Ocullatiux_sagi}

Giampaolo Proni | Chiome a confronto | DOI: 10.12977/ocula2019-14

no l'oggetto dell'indagine ${ }^{3}$. I giudizi e pregiudizi dell'analista sono analoghi a quelli dell'etnografo che si accinge ad accedere al campo. Negli studi qualitativi sui testi, o analisi desk, i dati reali usati dal ricercatore sono il significato che estrae dai testi, e quel significato è il prodotto della sua lettura, intendendo con questo termine qualsiasi tipo di fruizione di un testo. Un testo, naturalmente, può essere un documento scritto o un dipinto, un brano musicale, un film o qualsiasi altro sistema di segni con confini rilevabili e una forma sufficientemente stabile nel tempo. Il testo analizzato deve essere condiviso con i lettori affinché possano confrontarlo con il proprio punto di vista. I dati sui nostri oggetti di ricerca - le effettive acconciature di Trump e Kim - possono essere ottenuti dalle immagini e dalle descrizioni verbali in misura affidabile, in quanto consentono nel complesso (incrociandole e comparandole) una lettura sufficientemente coerente del loro oggetto semiotico e sufficientemente validata in relazione alle fonti (fotografie e video di autori e organizzazioni diverse). Perciò abbiamo dati adeguati alla costruzione di un campione testuale e possiamo iniziare l'analisi. ${ }^{4}$

Come riferimento per l'immagine di Kim consideriamo quella del giugno 2018, adottata per l'incontro con il presidente degli USA, ma è utile considerarne l'evoluzione (vedi TC-13 e in particolare TC-10).

\section{Traslazione di un testo da una cultura a un'altra}

La mia analisi è consapevole dei limiti nell'interpretare l'immagine di Kim all'interno della sua cultura ${ }^{5}$. Perciò mi limiterò alle ipotesi più solide e semplici.

3 I siti web contenenti le immagini e i testi usati per descrivere l'oggetto (o produrre una parte del testo) della presente ricerca sono elencati e numerati nella sezione Testi Campione (TC-nn), in fondo all'articolo. Nei Riferimenti si trovano altre fonti relative ai due personaggi analizzati e in particolare alla cultura e al sistema sociale e politico della Corea del Nord, meno noto al lettore occidentale di quello degli Stati Uniti. Wikipedia è stata usata come fonte primaria per una prima informazione $\mathrm{e}$ per la bibliografia.

4 Una ricerca sulle acconciature delle donne occidentali allo scopo di analizzare i valori sociali ad esse associati è stata proposta da McMurtrie (2010). Il quadro teorico è comunque diverso da quello adottato in questo caso.

5 In questo ambito sono fondamentali nel campo semiotico gli studi di Jurij Lotman (vedi Lotman 1975). La teoria di Lotman tuttavia non è di immediata applicazione all'analisi della 'mediosfera' attuale. La lingua naturale difficilmente si può oggi considerare il sistema modellizzante primario. La stessa idea di una gerarchia dei sistemi modellizzanti è dubbia. L'esposizione rapida e spezzettata dell'audience a un sistema mediatico multicanale, la prevalenza crescente delle immagini, la riduzione e a volte la quasi scomparsa della parola scritta, sono tra i fenomeni che testimoniano di una modalità di modellizzazione più complessa. Quanto al problema dell'influenza del filtro culturale dell'osservatore/analista nella traduzione da una cultura a un'altra, esso è oggi ben evidente. Si può dire che il '900 nella sua interezza è attraversato dalla riflessione sull'influenza dell'osservatore (e di tutto ciò che porta con sé) su ciò che è osservato, a partire dal principio di indeterminazione di Heisenberg. Ma nella pratica analitica la percezione e rimozione dei propri pre-giudizi resta difficile non solo perché molti di essi sono inconsapevoli, ma soprattutto perché è evidente che un'osservazione priva di riferimenti soggettivi è un'opzione puramente teorica. La soluzione più semplice è nella costituzione di team o reti multiculturali di analisti che condividano 


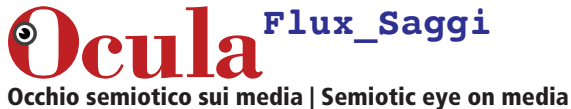

Giampaolo Proni | Chiome a confronto | DOI: 10.12977/ocula2019-14

Il problema prospettico si evidenzia nel momento in cui una cultura diventa consapevole che le altre culture hanno altri punti di vista, e che, poiché i significati sono funzione dei diversi punti di vista, se non si può ricostruire un altro punto di vista non si può ricostruire correttamente il suo universo di significati o enciclopedia. Concepire razionalmente che in una cultura si possano avere canoni estetici diversi dai nostri è relativamente facile, ma è assai difficile ricostruire il complesso di emozioni e sensazioni estetiche connesse a tali canoni. Tuttavia è proprio su queste percezioni che l'universo estetico si basa, non su una definizione formale. Una grammatica delle acconciature, che le riduca a elementi di articolazione inferiore e ad ognuno di essi associ un valore estetico codificato (per esempio "Il rosso Tiziano piace moltissimo quest'anno!"), non spiega quale specifico insieme emotivo-percettivo costituisca il valore estetico. Per fare un altro esempio, è noto che il gusto italiano non privilegia particolarmente (rispetto ad altri) il sapore agrodolce. Ora, è evidente che in tutte le culture del gusto si definisce e si sa riconoscere l'agrodolce. Tuttavia, lo specifico valore di gradevolezza di una porzione di crauti non è questione di comprensione linguistica, bensì di formazione culturale, forse con qualche substrato genetico (pare che questo accada per esempio con l'alcool, gli enzimi preposti alla digestione del quale non sarebbero presenti in misura uguale in tutte le etnie, determinando forse anche variazioni nel gusto $^{6}$ ). Questa sorta di semiotica di secondo livello risulta impossibile da acquisire come una semplice conoscenza, ma ha più la natura di un abito ${ }^{7}$.

\section{L'acconciatura come elemento del vestito}

La pettinatura, ovvero un modo artificiale di portare i capelli, è un elemento del vestito. Vestito, nelle scienze sociali, fa riferimento a ogni oggetto indossabile o alle modificazioni corporee adottate da un individuo nel suo comportamento in pubblico (Kawamura 2011: 7-8). Tatuaggi, monili, chirurgia estetica, calzature, biancheria, profumo, ecc., sono tutti elementi del vestito. Il vestito, a sua volta, è parte della più ampia categoria del comportamento di presentazione di sé, il quale comprende le norme del parlare, i codici del pudore e le buone maniere, comportamenti di ruolo e status symbols, ecc. ${ }^{8}$

Per quanto riguarda la semiotica, è stato Roland Barthes il primo a propor-

un metalinguaggio, in questo caso semiotico. Su Lotman vedi anche Traini (2005 e 2013, cap. 6).

6 Vedi Wikipedia, Alcohol tolerance, <https://tinyurl.com/y8sgdzzu>.

7 Il termine è usato nel senso di Peirce, vale a dire un modello di risposta cognitiva o comportamentale che costituisce il pieno sviluppo del significato secondo la definizione pragmaticista (vedi Peirce c.1907). Le opere edite e un'ampia selezione di manoscritti sono raccolti in Peirce 1931-1958 e Peirce 1982-in corso, e di norma ci si riferisce a queste fonti. La prima è tradizionalmente citata col formato: CP numero volume.numero paragrafo (es. CP 5.234) e la seconda con il codice: WR numero volume.numero pagina (es. WR 3.266). Vedi Riferimenti.

8 La letteratura in questo campo è ampia, non tanto in semiotica quanto in sociologia. Erwin Goffman può essere considerato l'iniziatore della ricerca su come le persone recitano i loro ruoli sociali, con il suo lavoro pionieristico The Presentation of Self in Everyday Life (Goffman 1956). 
Giampaolo Proni | Chiome a confronto | DOI: 10.12977/ocula2019-14

re di applicare le categorie linguistiche all'abbigliamento, elaborando alcune osservazioni di Nikolaj Trubeckoj e inquadrandole nella teoria del segno di Saussure $^{9}$. Nella trasposizione di Barthes al comportamento vestimentario, la langue corrisponde al costume come insieme di regole socialmente codificate del vestire, e la parole al singolo atto di abbigliamento. Il vestito è dunque una categoria che si suddivide in costume e abbigliamento.

Il costume è il sistema di norme che prescrivono, vietano, raccomandano o disapprovano maniere, tipi e caratteristiche del vestito.

L'abbigliamento consiste dei comportamenti vestimentari individuali adottati dalle persone. Alcuni aspetti del vestito appartengono dunque all'abbigliamento, come i tratti individuali o il fatto che una persona sia sciatta, o l'abitudine di un uomo di rimboccare le maniche della camicia. Nel passaggio citato da Barthes, Trubeckoj, come esempio di abbigliamento (cioè parole) riporta "le dimensioni individuali di un vestito e il suo grado di usura e di sporcizia" (Barthes 2006: 33). Saranno allora atti di abbigliamento anche gli opposti della sciatteria, vale a dire l'affettazione, l'eccesso di cura, il dandismo, le idiosincrasie: in generale qualsiasi variazione individuale della presentazione di sé.

\section{Trump e Kim: costume o abbigliamento?}

A prima vista, casi come quelli di Trump e Kim sembrano dunque casi di abbigliamento, perché sono variazioni individuali che non diventeranno mai tendenza e norma. Non vi è traccia di imitazione e nessun Trump-style o Kim-style si sta diffondendo o guadagnando seguaci. Tuttavia quando i soggetti sono rilevanti a motivo del loro ruolo sociale e mediatico, anche se trattiamo un caso di abbigliamento, esso acquista le caratteristiche di esempio, cioè di un simbolo ${ }^{10}$ e come tale ha carattere di regola, ha tratti concettuali. Ovviamente le categorie classificatorie di Peirce vanno considerate (e questo è presente anche nel loro stesso inventore, anche se non così chiaramente espresso) sempre come compresenti nella maggior parte dei casi. Icone, Indici e Simboli 'puri' sono rari perché entità logico-semiotiche in un certo senso 'estreme'. In questo caso, l'immagine pubblica di un capo di stato è un testo complesso, non si può paragonare a un simbolo logico o matematico come ' $x$ ' nella proposizione "sia x un numero intero". Tuttavia, resta il fatto che la relazione del segno con il suo oggetto (i valori ai quali esso fa riferimento) è convenzionale, e l'interpretante prodotto sintetizza la relazione correlando oggetto dinamico e contenuto del segno. In sintesi: Trump simboleggia gli Stati Uniti in quanto presidente eletto secondo le storiche regole e secondo gli

9 Nikolaj S. Trubeckoj, Principes de phonologie, Paris, 1949, citato in Barthes (2006: 33).

10 Il simbolo, nella semiotica interpretativa, è un tipo di segno che si riferisce al suo oggetto per mezzo di una regola, definita codice. Quando diciamo che una persona è un simbolo (come un re o un leader religioso) diciamo che, al di là della propria individualità, rappresenta dei valori generali, come una nazione, una visione morale, ecc. Questi valori esistono e sono definiti indipendentemente dalla persona che li incarna e possono incarnarsi in un un'altra persona o evento. 


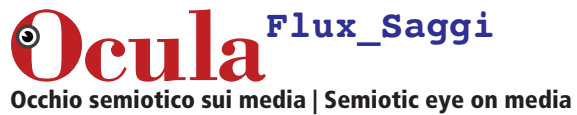

Giampaolo Proni | Chiome a confronto | DOI: 10.12977/ocula2019-14

storici valori americani ecc ecc, ma è comunque Trump, diverso da Clinton o da Bush, e questa parte è fortemente indicale. Le immagini di Trump e Kim, comunque, possono essere considerate istanze di costume e adatte a un'analisi semiotica.

\section{Livello espressivo}

Al primo punto dell'analisi troviamo la domanda sulla formazione del testo a livello espressivo: come sono costruite materialmente le pettinature dei nostri due leader?

\subsection{Trump: l'enigma del riporto}

Dalle immagini e video reperiti sul web e sulla stampa, è difficile comprendere come Donald Trump dia forma alla propria chioma. Diversi osservatori hanno ipotizzato, per esempio, che possa usare una parrucca o che si sia sottoposto a un trapianto. Niente di tutto questo. I capelli di Trump sono sottili e mostrano una stempiatura avanzata, ma sono autentici. Su questo punto ha fornito prove inconfutabili, sottoponendosi al "test del secchio ghiacciato" e permettendo a Jimmy Fallon di spettinarlo (TC-04 2016). Il colore della capigliatura, invece, è artificiale: un confronto tra foto più e meno recenti mostra come sia i capelli sia le sopracciglia siano tinti (TC-02 2015a).

L'acconciatura di Trump è uno stile di hair dressing e, a quanto risulta, Trump si pettina da solo. Tuttavia non si può parlare di semplice riporto, dato che non copre una calvizie ${ }^{11}$. È comunque costruita come un riporto, ma di un tipo particolare. Secondo l'interpretazione più valida che ho trovato (di uno stilista che ha chiesto di restare anonimo):

To me, it looks like a case of the classic old 'double combover'. I would hazard a guess that Donald's daily hair styling routine involves some rigorous back combing, followed by teasing hair from the left of his head over to the right side - then, teasing sections from the back over the front, finishing off with an Ozone-busting fix of industrial strength hairspray" (TC-o6 Page 2014).

Per quanto riguarda il livello espressivo del testo, quanto abbiamo trovato si può considerare sufficiente al prosieguo dell'analisi: abbiamo un'idea abbastanza chiara di come sia composto il nostro testo, di come rinvii al suo oggetto e di quest'ultimo in quando insieme di eventi empirici.

\subsection{Kim: dinastie comuniste e pettinature}

Sul piano espressivo, la pettinatura di Kim presenta un tratto tradizionale delle acconciature comuniste euro-asiatiche, cioè la pettinatura all'indietro. Da Karl Marx i leader comunisti ereditarono le idee ma non la capigliatura, che influenzò più gli anarchici, primo Bakunin.

${ }_{11}$ Ho proposto l'analisi semiotica del riporto in Proni (2001). 


\section{Ocullatiux_sagi}

Giampaolo Proni | Chiome a confronto | DOI: 10.12977/ocula2019-14

Lenin poté fare poco con i suoi capelli, e dunque il primo leader comunista che promosse un hairstyle e un outfit personale fu Stalin. Le tecniche di stampa e il culto della personalità diffusero la sua immagine in tutto il mondo. Stalin si pettinò sempre, in età adulta, con la fronte scoperta, i capelli all'indietro e senza riga. La pettinatura di Mao Zedong si può in qualche modo assimilare a quella staliniana. Nonostante l'amplissima stempiatura, il Grande Timoniere pettinava i capelli all'indietro e li radeva ai lati fino a sopra le orecchie. In più, Mao in molti ritratti mostra di far crescere fino a una certa lunghezza $i$ capelli ai lati, creando così due rigonfiamenti caratteristici (vedi Wikipedia, Mao Zedong). È questo il modello dell'acconciatura che i Kim si sono tramandati per tre generazioni. Se infatti cerchiamo foto di Kim Il-sung, il nonno del leader attuale, vediamo che copia quasi fedelmente lo stile di Mao. Pettinatura all'indietro, nessuna riga, senza basette, capelli mai al di sotto delle stanghette degli occhiali (TC-14, 15). Kim Il-sung lasciò il potere al figlio Kim Jong-il, che rappresenta la fase più artistica della dinastia e questa disposizione emerge anche nella sua acconciatura ${ }^{12}$. Jong-il ${ }^{13}$ adottò lo stesso taglio del padre come base, lasciando però crescere i capelli nella parte superiore della testa. In alcune foto pare persino che adotti la cotonatura per dare maggior volume. 亡̀ anche possibile che li tingesse di nero (vedi TC-16).

Trattando dell'immagine della famiglia Kim è importante anche il sintagma completo: acconciatura e abbigliamento. L'abito scelto da tutti e tre per le cerimonie ufficiali è una variante del cosiddetto 'Mao suit', il completo giacca e pantaloni indelebilmente associato a Mao Zedong. È evidentemente una diretta ispirazione ideologica a un leader molto importante nella storia della Corea del Nord . A sua volta Mao riprendeva uno degli abbigliamenti preferiti di Stalin. Si tratta in effetti di un capo di tipo militare, derivato da quella che i russi chiamavano giacca French (dal nome del comandante in capo della forza di spedizione britannica nella I guerra mondiale feldmaresciallo John French), adottata anche da Kerensky, Lenin, e Sun Yat Tsen ${ }^{14}$. Divenne però famosa come uno dei simboli della Cina comunista ${ }^{15}$. Questo abito è identico nei leader supremi e nei gradi inferiori dell'apparato politico.

La differenza tra i tre Kim sta nelle caratteristiche somatiche, nel modo di dare volume ai capelli e nella rasatura delle tempie, che raggiunge il massimo in Jong-un (vedi TC-10-13). Questa rasatura molto alta confina la capigliatura alla sommità del capo ${ }^{16}$. È un tratto espressivo ereditario, che varia progressi-

12 Era notoriamente un appassionato di cinema, al punto che fece rapire una nota attrice Sudcoreana per rilanciare l'industria nazionale (Little, B. 2018), e si interessò molto di arte (vedi Wikipedia Mansudae Art Studio).

${ }^{13}$ Come in altre culture asiatiche, anche in Corea il nome di famiglia precede quello personale.

14 Vedi Wikipedia, French (tunic) e ib. Mao suit e Stalin Tunic. A volte l'uniforme di Stalin viene erroneamente assimilata alla camicia tradizionale russa ripresa da Lev Tolstoj.

15 Anche Ho Chi Minh si ispirò a questo abito, adattandolo al clima del suo paese e usando preferibilmente colori chiari.

16 È quasi un mistero il retro della capigliatura di Kim Jong-un. Non ho trovato nel web nessuna foto che lo ritragga alle spalle. Secondo quando riporta Halloway 


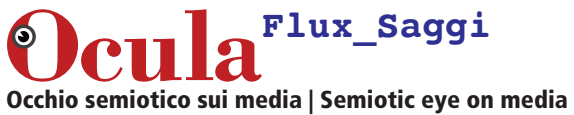

Giampaolo Proni | Chiome a confronto | DOI: 10.12977/ocula2019-14

vamente: aumenta dal nonno al padre al nipote ${ }^{17}$. Lo stesso si può dire di un altro tratto fisiognomico fondamentale, la pienezza del viso. Tutti e tre i Kim presentano tratti floridi, ma anche questo aspetto si espande nelle tre generazioni, arrivando in Jong-un a una rotondità apprezzabile, così come la sua intera costituzione è decisamente sovrappeso.

Un altro tratto è visibile in alcune fotografie: Kim Jong-un utilizza in certi casi della cera o gel per capelli per tenere la capigliatura a posto e darle volume e lucentezza (vedi TC-12).

L'immagine complessiva di Kim Jong-un rispetta un'iconografia fortemente ribadita dal sistema socio-culturale nord coreano: i ritratti dei primi due leader sono onnipresenti e costituiscono il centro dell'immaginario visivo mediatico, educativo, domestico e politico. Quanto a Jong-un, la sua apoteosi sembra ancora nella fase iniziale di un percorso articolato di successive e progressive enunciazioni visive (vedi BBC 2018).

\subsection{La dimensione paradigmatica}

L'imitazione è un comportamento che ha attratto l'attenzione dei sociologi fino dal XIX secolo (vedi Spencer 1896; Tarde 1890). Considerata sul piano espressivo, l'imitazione consiste nella selezione da un sistema paradigmatico di uguaglianze e differenze. L'imitatore seleziona alcuni tratti dal repertorio dell'imitato (che sia un individuo o un modello), e li adotta, sulla base di possibilità, opportunità, valutazioni di vario tipo.

Kim e Trump adottano entrambi varianti individuali di immagine, ma in meccanismi socio-semiotici diversi.

Kim imita la pettinatura dei suoi predecessori con variazioni personali. Come nel caso di Trump non possiamo parlare di tendenza, cioè un tratto di stile adottato consapevolmente da un numero crescente di persone. Secondo alcuni giornalisti Kim addirittura proibisce, sia pure in modo indiretto, di copiare la sua pettinatura (Wood 2017).

Trump è una celebrity, dunque un personaggio mediatico, caratterizzato da un look riconoscibile e unico, pur all'interno di un paradigma, quello dell'uomo di potere occidentale ${ }^{18}$.

Le scelte dei nostri due personaggi, quindi, si devono interpretare nel contesto di due società molto diverse. Quella di Kim è una società non-borghese, nella quale le norme e le regole sociali sono quasi tutte espresse da leggi o da ordini dello stato/partito/leader. Ufficialmente non vi sono classi, ma le diverse posizioni gerarchiche, occupazionali e persino il luogo di residenza sono,

(2018) nel commento di una foto (photo-gallery n. 24), fotografare una statua dei Kim alle spalle è considerato scortese.

17 Nella documentazione fotografica reperita sul web vi sono alcuni esempi del taglio di Kim Il-sung che mostrano una sfumatura ugualmente alta, ma sono più rare (vedi TC-15). Il nipote presenta questo tratto in modo costante.

18 La pettinatura di Donald Trump ha un rapporto curioso con quella di un altro presidente degli USA: John F. Kennedy. In un certo senso lo stile di Trump è una deformazione, quasi una caricatura di quello di J.F.K, che però divenne un vero modello di taglio maschile. Vedi Cool Men's Hair (s.d.). 
Giampaolo Proni | Chiome a confronto | DOI: 10.12977/ocula2019-14

a quanto si riesce a sapere, severamente regolate dallo stato/partito (Collins 2012).

Il sistema politico coreano rappresenta una singolare variante di quel 'culto della personalità' che nacque nei regimi comunisti, e che in Corea del Nord si unisce a elementi tipici della monarchia quali l'ereditarietà della carica ${ }^{19}$. La posizione di Kim Jong-un è quindi di leader unico e assoluto, e in quanto tale distinto da ogni altro individuo del suo Stato. È dunque impossibile essere come lui e aspirare a diventarlo.

La società di Trump è invece, al confronto, un fluido ribollente, nel quale lo stesso capo di Stato, mentre esercita le proprie funzioni e usa i propri poteri, è sottoposto a critiche, inchieste, contestazioni; la sua posizione è temporanea, la sua famiglia è inglobata nel circo mediatico, ogni suo commento e battuta si diramano in tempo reale nella rete globale. La società occidentale, soggetta a una forte pressione egualitaria e a una continua messa in discussione delle leadership, sembra quasi richiedere ai suoi politici una 'banalizzazione della personalità'. I leader occidentali dedicano sforzi costanti ad apparire 'cittadini come gli altri', a mostrare 'una famiglia normale', a esibire dichiarazioni dei redditi il più modeste possibile. Anche in questo Trump si distingue, ma sempre all'interno di un paradigma consolidato.

In questo modello la singolarità di Trump sta in una sorta di rifiuto di interagire, un isolarsi nel proprio spazio semiotico, in una sfera limitata alla propria persona. Anche se Trump si libera dalla pressione conformista del tipico politico democratico, non riesce però a diventare un modello, restando solo un caso originale, testardo propugnatore di un individualismo idiosincratico e privo di appeal, non in senso politico (dato che comunque ha milioni di elettori) ma proprio nel non voler ricercare il compiacimento estetico del pubblico. Per usare un termine affibbiato negli anni scorsi a Francesco Rutelli, Trump è un 'anti-piacione'.

Oggi, chi ha capelli sottili e radi può irrobustirli o ricostruirli con un trapianto, come Silvio Berlusconi, o mascherare la sua semi-calvizie con un semplice riporto come il Principe Carlo d'Inghilterra, o scegliere una virile rasatura come Bruce Willis. Persino la tinta scelta da Trump (estesa alle sopracciglia) sembra piuttosto eccentrica: è un colore che gli americani hanno definito "crusca di grano" ("wheat chaff"), raramente usata per sembrare biondi perché eccessiva. C'è qualcosa di iperbolico nelle scelte di Trump che rende la sua pettinatura un po' troppo vistosa. Questa caratteristica scoraggia e depotenzia la possibile imitazione nei suoi confronti e lo rende inadatto come modello estetico.

Se Trump in un certo senso non vuole, o non cerca di essere un model-

19 Vedi Wikipedia Cult of personality, North Korean cult of personality $e$ Constitution of NorthKorea. L'ereditarietà della carica di Leader Supremo non è ovviamente prevista dalla costituzione dello Stato. Tuttavia, nel preambolo ad essa, i primi due leader sono espressamente citati, Kim Il-sung come fondatore dell'ideologia Juche, sulla quale si basa lo Stato, e il secondo come suo prosecutore. Il culto della personalità è invece sancito da numerose leggi e regolamenti, in genere relativi ai primi due Kim. I casi di 'dinastie' democratiche non mancano, si veda quella dei Bush negli USA o dei Trudeau in Canada, ma non sono di solito sequenziali. 
Giampaolo Proni | Chiome a confronto | DOI: 10.12977/ocula2019-14

lo, Kim invece ha un diverso rapporto con la relazione di imitazione. Da una parte, copiare troppo pedissequamente la sua immagine è considerata una mancanza di rispetto e non viene incoraggiata ${ }^{20}$. I sosia e gli imitatori fanno quasi sempre satira politica e questa non è in genere ben vista nei regimi comunisti. Dall'altra, il ruolo del Leader Supremo è anche quello di modello di comportamento virtuoso, e certi parametri della sua immagine sono linee guida inderogabili per il cittadino modello. Inoltre, è del tutto normale in Corea del Nord che il regime dirami direttive sul corretto taglio di capelli (vedi BBC, 2005). L'imitazione delle pettinature dei grandi personaggi politici, spontanea o 'spinta' dai mass media, è diffusa in tutti i tempi e i paesi, basti pensare al taglio "all'umberta", ma solo in certi regimi si esercita pressione perché venga adottata, fino a emanare leggi suntuarie, come l'obbligo dell'acconciatura mancese in Cina (vedi Wikipedia Coda [acconciatura]).

Imitare Kim in modo smaccato, dunque, non è probabilmente una buona idea per un coreano. Prenderlo a modello è invece consigliato.

Fuori della Corea del Nord, invece, l'immagine di Kim non è considerata particolarmente desiderabile, e non registra casi diffusi di imitazione spontanea.

\section{Livello del contenuto: la funzione fatica del vestito}

Che cosa significano le acconciature di Kim e Trump? Cosa possiamo dire del livello semantico? ${ }^{21}$

La pettinatura è una parte dell'immagine pubblica di un individuo. È governata dalle norme socioculturali di presentazione di sé e tuttavia aperta a variazioni personali. Quanto a queste norme, la maggior parte di esse ha la funzione di organizzare la nostra apparenza pubblica in modo che sia coerente con il nostro corpo, il sistema sociale e le circostanze nelle quali siamo coinvolti. Così come c'è una grammatica del linguaggio parlato, c'è una grammatica del comportamento in pubblico (vedi Goffman 1963: 33-34). L'immagine pubblica di un individuo, come spettro semantico, è più limitata del linguaggio verbale, ma costituisce una condizione essenziale per la comunicazione ${ }^{22}$.

20 Si veda Halloway (2018) che formula per la verità entrambe le ipotesi (obbligo e divieto di imitazione) ma senza tentare una sintesi. In Nord Corea è difficile pensare a iniziative promozionali come questa, che ha avuto luogo ad Hanoi:

$<$ https://tinyurl.com/vj7hcr5>.

${ }^{21}$ Per la teoria del significato si tenga presente l'approccio interpretativopragmaticista di Peirce e sviluppato da Eco, centrato sulla nota massima: "Consider what effects, which might conceivably have practical bearings, we conceive the object of our conception to have. Then the whole of our conception of those effects is the whole of our conception of the object" (Peirce 1878, in 1982 WR 3.266).

${ }_{22}$ L'immagine pubblica nell'interazione non è veicolata solo dal canale visivo. USA anche quello acustico (lingua, stile, idioletto, 'tone of voice', lessico, ecc.), olfattivo (odore personale, profumo) e tattile (stretta di mano, saluti, contatti ammessi, come in una metropolitana affollata, ecc.). “... interaction (that is, face-to-face interaction) may be roughly defined as the reciprocal influence of individuals upon one another's actions when in one another's immediate physical presence" (Goffman 1956: 8). Oggi, anche l'interazione indiretta per mezzo di apparati di comunicazione è spesso equiparata a quella in presenza, benché non sia esattamente la stessa cosa. 
Giampaolo Proni | Chiome a confronto | DOI: 10.12977/ocula2019-14

La prima funzione dei complessi elementi testuali che formano l'immagine pubblica è fatica (pr. fàtica), vale a dire mostra come la persona si relaziona alle norme di quella specifica cultura e situazione ${ }^{23}$. Qualsiasi sia la ragione, ignorare le regole di presentazione di sé pone la persona fuori del gruppo sociale che le condivide. Tra i significati più importanti richiesti dall'interazione sociale vi sono quelli che rassicurano che il soggetto è legittimato dal gruppo sociale: si tratta dunque di costruzione e conferma della phatic communion, la funzione fatica secondo Malinowski. Egli vede la comunicazione fatica come una funzione basilare della cultura umana, quindi molto comune, mentre Jakobson pare ritenere che, proprio perché così comuni e onnipresenti, le espressioni fatiche giochino un ruolo secondario. Jakobson infatti non attribuisce alla funzione fatica un rilievo semantico, considerandola meramente come il mantenimento tecnico di un canale di comunicazione, mentre Malinowski implica che il fatico veicoli importanti contenuti sociali. Sono probabilmente da attribuire alla sfera fatica la gestione delle cornici (frame) dell'interazione sociale, e le funzioni onnipresenti di accettazione dei codici di gruppo, anche quando scorrono sottotraccia, dunque aspetti fondamentali e significanti ${ }^{24}$. Pur senza usare il termine 'fatico', Goffman ha una posizione simile quando tratta del 'body idiom' (Goffman 1963: 35).

Barthes concorda su questa funzione del vestito, benché le dedichi solo alcune righe. Afferma che "L'indumento è infatti il significante di un solo significato principale, che è il modo o il grado di partecipazione di colui (gruppo o individuo) che lo indossa" (Barthes 2006: 22). La definizione esprime in termini socio-semiotici il concetto di "phatic communion". Barthes (come Jakobson) sottovaluta però l'importanza di questo tipo di significati e le sfumature che possono assumere. La ragione a mio parere è che Barthes, in quanto strutturalista, aveva sempre in vista il sistema linguistico, capace di produrre proposizioni e dotato di due livelli di articolazione. La sua idea era che i comportamenti sociali possono codificare contenuti articolati e funzionare come un linguaggio. Il campo più ampio e non formalizzato del comportamento in generale, nel quale è difficile trovare un codice analogo a quello della lingua, gli sembrava probabilmente un terreno troppo insicuro per la teoria saussuriana (cfr. Barthes 2006: 35). Infatti, dopo i primi tentativi di leggere il comportamento vestimentario, Barthes si concentrò sulle riviste di moda come materiale più adatto a studiare le ideologie sottostanti la società contemporanea.

Ritengo che l'approccio migliore sia considerare i comportamenti vestimentari nel loro funzionamento fondamentale come componenti della sfera fatica e della cultura materiale: da una parte essi contribuiscono ad adattare

23 La nozione di "phatic communion" fu sviluppata per la prima volta da Bronislaw Malinowski (1923), e successivamente ripresa e introdotta in linguistica e semiotica da oman. Jakobson (1960). Vedi anche Proni (2015).

24 La nozione di frame in questo articolo è tratta da Eco (1979: 20-21), che fa riferimento, come fonte e confronto, ai lavori di Winston, van Dijk, Petöfi e naturalmente di Peirce. Frame analysis di Goffman, del 1974, è elencato nella bibliografia di Eco ma non citato nel testo. Il concetto è stato introdotto per la prima volta nel libro del 1972 Steps to an Ecology of Mind di Gregory Bateson. 
Giampaolo Proni | Chiome a confronto | DOI: 10.12977/ocula2019-14

l'apparenza pubblica di un individuo o un gruppo alle variabili che lo descrivono socialmente (età, genere, reddito, stile di vita, ecc) e dall'altra esprimono l'uso personale del costume. L'immagine pubblica risulta in questo modello una sorta di superficie $3 \mathrm{D}$ plasmata da un lato dalle spinte endogene di tipo più o meno consapevole e volontario e dall'altro dalle spinte esogene di tipo ambientale, sociale e interpersonale. Semioticamente, le dinamiche che producono morfologicamente questo 'involucro' si possono esprimere come programmi narrativi modalizzati secondo il potere, volere, dovere.

Se seguiamo questa teoria, dobbiamo allora indagare quale modo di partecipazione significano le acconciature analizzate, vale a dire, come sono posizionate nel sistema di valori sociali relativi all'immagine pubblica.

Il sistema semantico connesso all'interpretazione di simboli ha tre principali componenti. Il primo è il significato socialmente codificato di un testo, e può essere descritto come l'interpretante standard o immediato. Il secondo è la relazione dinamica tra l'intenzione dell'enunciatore, ciò che vuole esprimere, e l'interpretazione dell'enunciatario, il significato pienamente sviluppato. Il terzo sono le conseguenze pragmatiche logicamente inferite dai passi precedenti. L'interpretazione è un flusso di segni: su questo Peirce è chiaro ${ }^{25}$.

Abbiamo già discusso del primo livello: vediamo un uomo con capelli gialli tinti e una strana e complicata pettinatura, riconosciamo il Presidente degli Stati Uniti; vediamo un giovane paffuto con un taglio curioso, riconosciamo il leader della Corea del Nord.

Consideriamo ora il secondo livello: le intenzioni e le interpretazioni comuni.

\subsection{La mascheratura di Trump}

È ovvio che chi usa il riporto e la cotonatura vuole mostrare capelli più folti. Il colore invece si applica per due motivi: per mantenere la tinta naturale man mano che i capelli ingrigiscono o per cambiarla. Le due cose si possono fare contemporaneamente ed entrambe vengono usate per far apparire qualcuno come non è. Trump perciò vuole apparire con capelli più biondi e più folti, benché non li abbia così. Questo è quanto possiamo dire in relazione alle intenzione coscienti.

Tuttavia, a questo punto sorge una domanda di importanza non secondaria. Il soggetto crede e richiede che le sue intenzioni siano intese come vere? La risposta più verosimile è no. È improbabile che Trump creda che il colore dei suoi capelli sia considerato naturale e l'evidente riporto come una vera capigliatura. Comunque, questo è del tutto normale: altrimenti avremmo assistito alla scomparsa dei capelli bianchi nei paesi industrializzati, principalmente nelle donne.

Maschere di questo tipo sono di fatto molto comuni, ed è necessario trattarne più ampiamente.

${ }_{25}$ Un'esposizione della teoria dell'interpretazione di Peirce si trova in Peirce (c. 1907). La mia sintesi qui è una semplificazione e un adattamento. 


\section{Ocullatiux_sagi}

Giampaolo Proni | Chiome a confronto | DOI: 10.12977/ocula2019-14

\subsection{Gli inganni palesi dell’immagine pubblica}

Il tipo di cura personale della quale stiamo parlando è, come altre, l'adozione di una simulazione nell'immagine personale che può essere definita con l'ossimoro inganno palese. Un inganno, per definizione, mira a confondere, a far parere vero ciò che è falso. Si assume così che tingere i capelli per nascondere la canizie sia fatto perché sembri vero. È ugualmente evidente, tuttavia, che non ci aspetta di essere creduti: l'inganno è debole, quasi sempre scoperto, rivelato. In alcuni casi questi limiti sono lampanti: nessuno oggi pensa che il rossetto sia un vero tentativo di inganno: le sfumature e il lucido che dona alla bocca non si trovano in natura. Il make-up consiste infatti per lo più nell'alterare i tratti naturali. Esso non reclama credibilità: è in parte imitazione e in parte ornamento ${ }^{26}$. Riguarda la gestione dell'apparenza. La presentazione di sé ha proprio la funzione (prettamente semiotica) di far apparire socialmente quello che il soggetto non è personalmente.

La parte del corpo scelta per l'operazione di mascheramento (capelli, sopracciglia, labbra, ciglia, ecc.) è manipolata secondo un continuum che va da un inganno più credibile (una tinta di capelli che imita il colore naturale o una chirurgia estetica che mira a rimuovere i segni dell'età, ecc.) fino ad alterazioni vistose, forme innaturali. In generale, tuttavia, il mascheramento credibile è di breve durata e di scarsa efficacia. La funzione estetica (qualunque possano essere i canoni di tale estetica) prevale: "Certo che i miei capelli sono bianchi", una signora matura comunica con il suo colore, "ma penso di sembrare meglio in questo modo", e le persone attorno a lei rispondono col proprio comportamento: "Lo sappiamo e stiamo al gioco, così come tu accetti la nostra apparenza". Il gioco cooperativo produce e mantiene quel 'livello di bellezza' collettivo che influenza in maniera positiva la sicurezza individuale dei membri del gruppo e mantiene un ambiente sociale percepito dai partecipanti come positivo o almeno rassicurante.

Dopo Goffman, la recita teatrale è una metafora stabilmente introdotta in sociologia (Goffman, 1956), e dunque tutti gli inganni palesi si possono vedere come recite che si svolgono all'interno di frames o canovacci più o meno codificati. In termini semiotici sono aspetti figurativi di narrazioni sociali prodotte secondo canoni ${ }^{27}$.

${ }_{26}$ Vi sono importanti esempi di make-up 'innaturale', come l'annerimento dei denti nell'antico Giappone, le tinte per capelli azzurre, rosa e verdi tanto in voga oggi, o le pratiche di allungamento del collo o delle orecchie. La relazione tra il naturalismo e l'artificiosità cospicua' nella presentazione di sé sarebbe molto interessante da analizzare. Le imposture anti-naturali sono correlate alle estetiche post-moderne, che criticano l'equivalenza di bellezza e natura, tipica delle estetiche precedenti. L'estetica pop, con l'esaltazione di volumi e colori, è una posizione intermedia: non è antinaturale ma al massimo iper-naturale. Per un approccio socio-psicologico al make-up vedi Di Donato (2015) inclusa la bibliografia.

${ }_{27}$ Vedi figurativizzazione e figurativo in Greimas e Courtés (1986). In Proni (2007) riporto i risultati di uno studio pilota sul campo che applicava lo schema narrativo al fashion shopping. 


\section{Ocullatiux_sagi}

Giampaolo Proni | Chiome a confronto | DOI: 10.12977/ocula2019-14

\subsection{Funzione e significato di Kim Jong-un}

7.3.1 Il regime nord coreano e l'acconciatura maschile

Riguardo l'aspetto semantico dell'immagine di Kim in Corea possiamo sicuramente affermare che si estende ben oltre l'apparenza pubblica. Il Leader Supremo o suryeong (vedi Collins 2012: 15-16) è al vertice dell'ordinamento statuale, al di sopra del partito e della costituzione, e il culto della sua personalità è stabilito per legge. La sua famiglia gode di una posizione unica e superiore a ogni altra. In particolare, i due primi Kim sono ritenuti esseri di suprema capacità, statisti di livello insuperabile. Sono rappresentati in statue colossali e omaggiati con cerimonie e celebrazioni. I loro ritratti devono essere presenti in ogni casa, su una parete a essi riservata, così come in ogni edificio pubblico, carrozze ferroviarie e della metropolitana, ecc., e sono previste ispezioni per verificare che siano tenuti in modo adeguato. Qualsiasi offesa, verbale o materiale, a queste icone è un grave reato. L'esposizione e il trattamento dei ritratti dei Kim sono soggetti a regole complesse, che mutano con una certa frequenza. I volti dei due leader supremi compaiono anche su un badge, che viene donato a ogni cittadino all'età di dodici anni e che deve essere appuntato sul cuore ogni volta che si esce di $\operatorname{casa}^{28}$. Kim a volte lo indossa a volte no. Queste icone ${ }^{29}$ hanno una posizione così centrale nel sistema espressivo e comportamentale dell'intero ambiente dei cittadini da dover necessariamente avere un valore semantico proporzionale.

Non sorprende dunque se neppure i capelli sfuggono al controllo del regime. Per esempio, nell'inverno 2004-2005 sui media nazionali fu lanciata una campagna contro i tagli non corretti. La televisione, sottolineando l'importanza di igiene e salute "showed various state-approved short hairstyles including the "flat-top crew cut," "middle hairstyle," "low hairstyle," and "high hairstyle" - variations from one to five centimetres in length" (BBC 2005).

In questo contesto si comprendono anche le letture giornalistiche più recenti di tagli di capelli 'vietati' e 'permessi' (Wood 2017) ${ }^{30}$.

Elementi di occidentalizzazione si stanno però manifestando nell'immagine di Jong-un, come l'abito a giacca che ha indossato in occasione del messaggio televisivo di capodanno e sono comunque significativi (Loomes 2019; Corriere.it 1/4/2018). Questa dinamica di immagine è sicuramente eccezionale nella storia della dinastia. Non vi è dubbio che, nel linguaggio non verbale, Kim Jong-un abbia manifestato una certa imitazione del costume occidentale, e lo stesso si vede nelle immagini che ci arrivano da Pyongyang ${ }^{31}$.

28 Vedi Wikipedia Kim Il-sung and Kim Jong-il portraits e Siddiqui (2018).

29 Il termine in questo caso va inteso in senso culturale di 'immagine mediatica esemplare'.

30 D’altra parte, anche in Europa e USA, quando scoppiò la moda dei capelli lunghi per i ragazzi, non si contarono le reazioni a volte anche violente. Si veda a esempio un articolo di P.P. Pasolini (Pasolini 1973).

${ }_{31}$ Secondo diverse fonti Kim ha incoraggiato una serie di cambiamenti nel consumo e nello stile di vita della Corea del Nord che sembrano andare nella direzione di un avvicinamento ai paesi asiatici più sviluppati, come la Cina (vedi Grappe 2018). Tuttavia pare che la maggioranza di questi sia limitata alla capitale, nella quale vive 


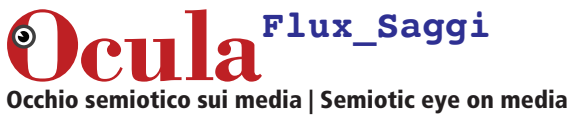

Giampaolo Proni | Chiome a confronto | DOI: 10.12977/ocula2019-14

L'immagine di Kim Jong-un nella sua cultura va analizzata in relazione a un sistema di valori rigido e onnicomprensivo, nel quale il leader supremo assume un valore simbolico altissimo. I cambiamenti della sua immagine non sono dunque scelte individuali, ma coinvolgono il sistema semiotico dello stato e della società nel suo complesso.

\subsubsection{Kim in occidente}

Kim Jong-un, quando si trova all'estero, sembra comportarsi come in patria. Tuttavia, il suo significato muta nettamente, perché si tratta di un testo ritagliato dal contesto abituale e trasportato in un altro. Da questa traslazione deriva l'effetto di estraneità che Kim suscita negli osservatori occidentali. È un essere supremo provvisoriamente traslato in un contesto nel quale il suo spettro semantico abituale è troncato.

Le immagini ufficiali delle apparizioni pubbliche di Kim Jong-un in Corea sono per noi narrazioni quasi umoristiche, caricature di regimi comunisti ormai scomparsi. In molte foto compaiono alti ufficiali o funzionari che prendono rispettosamente appunti mentre il leader supremo parla, per evitare di perdere anche una sola delle sue preziose parole (vedi Wuyiran 2018). Di fatto, si tratta di una forma rituale, che testimonia uno dei tanti carismi del Leader Supremo, quello di saper dare istruzioni e insegnamenti su ogni aspetto della vita e del lavoro.

Il servizio sulla partenza del treno da Pyong Yang verso Hanoi per l'incontro con Trump è particolarmente divertente (BBC 2019b). L'entusiasmo del popolo in presenza di Kim è ovviamente sempre vivissimo, ma gli occhi occidentali tendono sempre a cogliere l'opposizione spontaneo vs obbligato, e la marca figurativa della spontaneità è per noi il disordine e la differenza. Esultare collettivamente e ordinatamente per un occidentale equivale a fingere. Allo stadio le cheer leaders che eseguono le loro coreografie, al nostro sguardo, si distinguono dall'esultanza dei tifosi. È evidente dunque che il significato che noi non-coreani possiamo attribuire a queste scene è diverso da quello che propriamente rivestono nel loro contesto.

Tuttavia, anche ai nostri occhi la dinastia esprime qualcosa con le differenze di acconciature dei suoi esponenti: Kim Jong-il, per esempio in qualche modo adattò la sua immagine allo zeitgeist, lasciando i capelli un po' ribelli sul capo, dedicandosi alle arti, in particolare al cinema.

Il faccione paffuto e la pettinatura di Jong-un ci sembrano bizzarre e buffe, mentre una tale percezione, per un nord coreano, costituirebbe un grave travisamento. Sarebbe come se un cattolico percepisse il papa come un vecchietto in tunica e zucchetto bianchi.

\subsubsection{Kim: la narrazione dell'uguaglianza}

L'inganno palese più comune nella società occidentale riguarda aspetti estetici individuali, quasi si cercasse un'omologazione del corpo in una cultura dell'individualismo economico e psicologico.

la parte favorita della popolazione. Poiché lo stato e il partito possono decidere dove una famiglia risiede, di fatto ogni provincia del paese è destinata a ospitare un certo segmento della popolazione (vedi Collins 2012). 


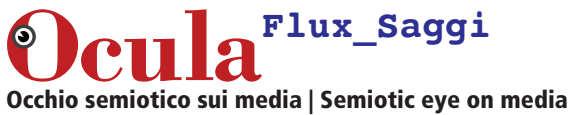

Giampaolo Proni | Chiome a confronto | DOI: 10.12977/ocula2019-14

I regimi comunisti hanno invece esteso all'intero apparato del potere l'obbligo di un'immagine egualitaria. Kim appare in diverse foto al fianco di altri uomini con un abito identico, probabilmente alti funzionari dell'apparato del partito o dello stato.

Anche il vestito occidentale maschile, l'abito a giacca, definito power suit, è egualitario ${ }^{32}$ ma il vestito dei leader comunisti ha una marca semantica in più: è sostanzialmente una divisa, sia pure di un esercito popolare. Nel codice vestimentario della Corea del Nord, l'abito ‘alla Mao' (sia pure corretto) si contrappone alla divisa dei militari (piena di decorazioni negli altri gradi) come una sorta di 'divisa civile'.

Il codice che troviamo in Nord Corea è dunque diverso dal costume del potere adottato da Trump e da molti occidentali. Quello che si narra è un'uguaglianza ispirata ai principi della società senza classi, morale, rigorosamente egualitaria. I valori semantici più evidenti sono: esercito popolare, uguaglianza, praticità, rifiuto di ogni lusso, parsimonia, negazione dell'individualismo.

La finzione dell'abito di Kim è evidente quanto il riporto e la tinta di Trump: i privilegi del leader supremo rispetto ai suoi cittadini sono superiori a quelli (già notevoli) che separano il milionario-presidente dall'americano medio $^{33}$. L'intera società nord coreana è strutturata in un sistema di gruppi sociali distinti per diversi gradi di privilegio sociale, economico e giuridico (vedi Collins 2012; Wikipedia [2019], Songbun ${ }^{34}$ ).

Ancora una volta, abbiamo la conferma che l'immagine pubblica è una rappresentazione complessa nella quale sono molti gli aspetti finzionali, spesso tesi a correggere o alterare anche in modo macroscopico lo status sociale effettivo del soggetto.

In questo modo un leader può apparire allo stesso tempo parte del popolo e porsi al vertice supremo del potere.

7.3.4 La circolazione dei valori estetici in due diversi sistemi

Le analisi della pettinatura di Kim Jong-un e di Donald Trump a questo punto divergono. Mentre Trump si distingue per un idioletto estetico individuale, Kim rappresenta un'estetica dinastica e ufficiale; il linguaggio che usa è ampiamente condiviso.

Sono inoltre diversi i paradigmi sociali che regolano la circolazione delle forme estetiche nelle due culture.

Nella cultura occidentale contemporanea i canoni di bellezza sono sempre imitabili, e molti di essi sono acquistabili sul mercato. È per noi impensabile che le acconciature di celebrity come Elvis Presley o Bob Marley non diventino un modello che si diffonde. Abbiamo dunque una mobilità di modelli (il

32 L'omologazione dell'abito occidentale maschile ufficiale è stata letta come 'grande rinuncia' della borghesia, che contrappone la severità dei propri costumi all'esibizione e all'immoralità dell'ancien régime. La teoria ha origine in Flügel (1930) e viene poi ripresa da König (1973), con riferimento all'abito quacchero.

33 Sulle residenze di Kim Jong-Un e sui suoi treni personali vedi Wikipedia Residences of North Korean leaders, Ryongsong Residence e North Korean leader's trains .

34 Entrambe queste fonti riportano una bibliografia e sono consultabili online. 


\section{Ocullatiux_sagi}

Giampaolo Proni | Chiome a confronto | DOI: 10.12977/ocula2019-14

rock and roll, poi il pop, poi il punk e così via) e una mobilità di ruoli sociali (oggi presidente, domani ex-presidente; oggi studente, domani professionista o imprenditore; ecc.).

La vita del sistema nord coreano è invece governata da regole che costruiscono un paese simile a un apparato, una macchina organizzativa, che ruota attorno a una razionalità centrale, incarnata dal leader supremo e avanza verso un radioso avvenire sotto la sua guida. Questa organizzazione persegue un'efficienza orientata verso la lotta costante contro i nemici di classe, interni ed esterni, che precede ogni altro principio.

Restando a un livello di descrizione sommaria delle due società, è evidente che da una parte abbiamo mobilità, disordine ed entropia, dall'altra centralismo, ordine e comunicazione gerarchica nei processi. Posto al vertice di questo sistema, Kim Jong-un spicca per la sua solarità, il suo conformarsi ai dettami della dottrina vigente e allo stesso tempo adempiere alla propria funzione di supremazia, di superiorità personale. Tuttavia, mentre Kim è parte essenziale del sistema al centro del quale è collocato, e non avrebbe spessore semiotico senza di esso, Trump, proprio perché è temporaneo e limitato nei suoi poteri, mantiene una certa separatezza dal sistema in cui si colloca. $\mathrm{Cu}-$ riosamente, abbiamo da una parte un sistema impersonale che ruota attorno a una suprema personalità, dall'altra un sistema di individui indipendenti che pone al proprio centro un ruolo, una carica, che deve agire senza farsi influenzare troppo dalla propria soggettività.

\subsection{L’interpretazione personale delle fiction sociali: l’idioletto di Trump}

Continuiamo con il secondo livello dell'interpretazione, ma cercando di svilupparlo ulteriormente. Abbiamo visto che la pettinatura di Donald Trump ha caratteristiche di peculiarità e complessità espressiva, ma che non produce chiari effetti di senso. Lo stile di Trump è bizzarro senza essere trasgressivo: è peculiare, originale e abbastanza vistoso, ma non mira a particolari significati. Trump non è né un dandy né un alternativo ${ }^{35}$. Sotto questo aspetto lo stile del primo ministro greco Alexis Tsipras è più significativo; non portando la cravatta veicola di essere estraneo all'élite borghese, dunque adotta un codice condiviso (vedi BBC 2015).

A questo stadio dell'analisi ho avuto la sensazione che la pettinatura (come la cravatta $)^{36}$ siano molto importanti per Trump ma allo stesso tempo pare

35 Il dandy è un altro caso di individuo che costruisce la propria immagine come un idioletto individuale. Tuttavia il dandy non è avulso dall'estetica del suo tempo; al contrario, propone sé stesso come un interprete modello, e la sua immagine pubblica è un'opera d'arte. Solitamente disprezza i seguaci, e se il suo stile inizia a diffondersi lo muta rapidamente; tuttavia ama essere ritratto come arbiter elegantiarum. Lo stile del dandy è un idioletto temporaneo e un'opera d'arte: la sua immagine personale viene offerta, per essere decodificata, ai pochi che la apprezzano. Vedi Baudelaire (2004); Muzzarelli (2015).

${ }^{36}$ Lo stile di Donald Trump ha infatti un'altra particolarità: la cravatta. Viene tenuta molto lunga, fin sotto la cintura, così da coprire l'inguine quando è seduto. E Trump ha anche l'inesplicabile abitudine di fissare con nastro adesivo l'estremità stretta della cravatta a quella larga (vedi Christian 2017 e in particolare le immagini). 
Giampaolo Proni | Chiome a confronto | DOI: 10.12977/ocula2019-14

che non abbia interesse a rendere comprensibile agli altri questi significati ${ }^{37}$. Perché? E quale effetto di senso ne consegue?

Abbiamo detto che si tratta della variazione individuale di una narrazione finzionale. Ogni inganno palese ovviamente ha un codice. Questo codice normalmente non elenca tutte le possibili variazioni di un tratto, ma alcuni punti sono marcati lungo diverse dimensioni e si stabiliscono alcuni limiti. L'igiene personale, per esempio, è un obbligo forte nella maggior parte dei gruppi sociali: un uomo con una lunga barba è accettato, ma deve essere pulita.

La migliore spiegazione è probabilmente che l'acconciatura e altri dettagli del look di Trump producano questa sensazione perché sono espressi in un linguaggio parlato solo da una persona, cioè un idioletto.

Umberto Eco definisce l'idioletto come "[...] potenziale 'nuovo codice' [che] ha generato un solo testo ed è stato parlato da un solo mittente" (Eco 1976: 339).

Gli idioletti si trovano in diversi ambienti. Il più comune è la famiglia, specialmente quando vi sono bambini. È normale per i bambini coniare neologismi deformando le parole o inventando varianti immaginose. Esse vengono poi adottate dai genitori e/o condivise con i fratelli. Sono nomignoli di persone o animali, giocattoli o alimenti, o altri elementi della vita quotidiana. Il bambino piccolo è solo parzialmente consapevole di usare un idioletto. Accade che lo usi con estranei senza rendersi conto che non viene compreso. Un adulto, invece, non usa l'idioletto famigliare o di coppia con interlocutori che non lo capiscono. Fa parte della competenza di un parlante identificare l'area linguistica condivisa con l'interlocutore.

La questione nel nostro caso non riguarda però il contenuto bensì l'idioletto stesso: che cosa significa adottare un'alterazione individuale e idiolettale? In altri termini, consideriamo il significato metasemiotico di questa scelta particolare: non il significato dell'enunciato (cioè un uomo maturo con capelli tinti e cotonati) ma quello dell'enunciazione, cioè il medium semiotico e la sua occorrenza. Non quello che Trump dice ma il linguaggio che usa per dirlo.

Un altro punto da rimarcare è che, essendo un atto di cura di sé, quello di Trump è un idioletto estetico. Eco spiega le caratteristiche degli idioletti estetici, ma il suo interesse è limitato all'opera d'arte (Eco 1976: 338-341). Eco non prende in considerazione l'estetica della vita quotidiana, che è divenuta così importante a partire dagli anni ' $80^{38}$. In effetti, il look di Trump non ha alcun carattere di arte (non ha nulla di innovativo o creativo, come si è visto) ma ha un valore estetico, è un tratto di uno stile personale ${ }^{39}$.

Ora siamo in grado di dare una risposta: persistere in un idioletto estetico solitario e unico comunica che il soggetto adotta un paradigma individuale senza preoccuparsi se sia condiviso da un gruppo sociale anche ristretto. Se applichiamo la definizione di Barthes/Malinowski, Trump cerca di non esprimere il 'grado e modo di partecipazione', ovvero si sottrae almeno parzialmen-

37 Ne sono prove la cura che richiede la pettinatura e l'attenzione a nastrare le due parti della cravatta ogni volta che viene annodata.

38 Sulla riduzione dell'estetico all'artistico vedi Di Stefano (2012: 8-9).

$39 \mathrm{Su}$ questa giustapposizione vedi Matteucci (2016). 
Giampaolo Proni | Chiome a confronto | DOI: 10.12977/ocula2019-14

te alla 'phatic communion'. Trump, con la sua acconciatura e altri aspetti della sua immagine pubblica, come un bambino parla pubblicamente un idioletto personale.

\subsubsection{Il significato dei linguaggi}

Trump usa dunque un idioletto estetico non condiviso in una narrazione sociale finzionale o inganno palese, ma non si cura delle interpretazioni altrui, né comunica le proprie. Ha mostrato di essere consapevole della curiosità che i suoi capelli destano, e ha anche accettato di essere preso in giro (TC-04 2016, TC-o8 2014) ma non ha letteralmente cambiato un capello del suo look né ha mai spiegato le proprie scelte.

Situare se stessi al di fuori dell'asse trasgressione vs conformismo, trascurare (parzialmente, ma simbolicamente) di aderire alla phatic communion è possibile solo se si è così consapevoli dei codici correnti da creare un idioletto originale che sfugga all'opposizione o se si produce semiosi in un modo così individualistico e spontaneo perché si ignorano i codici sociali. Ci troviamo così sull'asse pianificazione vs spontaneità. Basandoci su quanto sappiamo, il caso più probabile è il secondo: lo stile di Trump è inintenzionale. È spontaneo. Trump è capace di un comportamento originale nel vestire, le sue idee estetiche sono peculiari, ma sono vaghe, non può dare loro un significato se non che sono personali, peculiari. È cosciente che la sua immagine personale è originale, ma non coglie pienamente come le altre persone la giudicano, $\mathrm{e}$ rifiuta di occuparsene ${ }^{40}$.

Questo si può spiegare con l'ipotesi di una scarsa abitudine al feedback. Pur entro i limiti di un comportamento normale, Trump mostra i sintomi di un lieve autismo nella presentazione di sé. La sua immagine non si relaziona del tutto con le tendenze, l'enciclopedia socio-estetica corrente. Considerando l'alta posizione che occupa, tuttavia, anche piccoli problemi possono avere conseguenze serie.

Se estendiamo l'ipotesi, possiamo supporre che Trump abbia difficoltà nel comprendere quello che le altre persone comprendono del suo messaggio. Ha un'idea imprecisa delle interpretazioni condivise nel suo gruppo sociale, e non è pienamente cosciente che lui, e lui solo, attribuisce certi significati a certe espressioni. In breve, pensa che gli altri possano trovare esteticamente positi-

40 Queste affermazioni non sono di ordine psicologico bensì derivano da inferenze basate sul macrotesto mediatico costituito dall'immagine e dalla comunicazione di Donald Trump. Che Trump sia consapevole che la sua immagine personale è ritenuta (per lo meno) originale si può ragionevolmente inferire dal fatto che, se così non fosse, sarebbe davvero difficile per lui spiegare perché così tanti messaggi mediatici prendono di mira la sua capigliatura. Il fatto che non dedichi però attenzione a questa stranezza percepita si spiega da una parte con l'assenza di qualsiasi accenno ad essa nei messaggi che emette (se non che si tratta dei suoi veri capelli e non di una protesi), e dall'altra con il fatto che non l'ha mutata nemmeno minimamente. Che questa indifferenza implichi anche una mancata comprensione del giudizio estetico altrui è ipotesi più debole ma non infondata, che si basa sull'estrema cura che Trump dedica alla propria immagine, proprio a partire dall'elaborata pettinatura, includendo la precisa lunghezza e la nastratura della cravatta, la tinta delle sopracciglia e dei capelli, l'abbronzatura. Parametri non estranei ai codici estetici del maschio americano WASP, incluso un controllo rigoroso dell'ordine personale. 
Giampaolo Proni | Chiome a confronto | DOI: 10.12977/ocula2019-14

vo quello che egli trova tale. Comunque, non solo non gli interessa verificarlo, ma probabilmente non è stato educato a farlo.

Questa forma di atteggiamento comunicativo rafforza il lato della produzione del discorso (il soggetto produce messaggi nei quale crede fermamente e che difende senza curarsi dell'opinione comune) ma indebolisce il lato della ricezione (il soggetto pensa che gli altri comprendano sempre ciò che intende dire, ma comprende solo parzialmente e con scarsa precisione ciò che comprendono davvero).

Questa configurazione è indubbiamente tipica di un certo infantilismo, e questo è coerente con lo sfondo biografico di una persona ricca che è nata e cresciuta in un ambiente come New York City, in un settore nel quale le relazioni sociali si stabiliscono entro una cerchia ristretta. Un tratto, se ci pensiamo, che certamente implica un po' di innocenza e semplicità, e che può essere apprezzato da un elettorato disgustato e disilluso dai politici tradizionali. L'estraneità di Trump ai segnali codificati della phatic communion può essere presa per un segnale di individualismo e autonomia dalle regole sociali, un valore tradizionale nella cultura degli Stati Uniti.

L'ingenuità può essere intesa come indice di sincerità. Un bambino può dire qualcosa che non comprendiamo bene perché adotta un idioletto famigliare, ma non dubitiamo della sua sincerità. Qualcuno che non riesce a capire se lo comprendiamo, cioè che non sa capire bene chi siamo, è sincero, perché non ha né il motivo né la capacità di ingannarci. Un truffatore è invece sempre molto attento a quello che le sue vittime intuiscono, comprendono e pensano. Che Trump finga questa ingenuità è sempre possibile, ma i tratti espressivi del suo look, così maniacali, la goffaggine con la quale sono tenuti nascosti (nascondere per rivelare), indicherebbero una maestria superiore ai vantaggi che ne potrebbero derivare.

\subsubsection{Kim: un costume dinastico}

Nel caso di Kim non possiamo parlare di un idioletto. L'immagine di Kim Jong-un si inserisce in quella della sua famiglia, e tutti e tre, come si è visto, seguono il costume della categoria, sia pure ridotta, dei leader rivoluzionari comunisti.

Allo stesso tempo, Kim usa la propria immagine per comunicare ed esprimere la sua modernità, la sua apertura al mondo ma, a differenza di Trump, non può essere soggetto a critiche mediatiche da parte di nessuno, nel suo paese. La sua immagine personale è l'enunciazione unica e suprema della direzione ideologica del sistema.

\subsubsection{Kim e l'estetica occidentale}

Visto in occidente, tuttavia, anche il look di Kim Jong-il è un idioletto, un linguaggio senza altri parlanti. Qualcuno forse sa che si tratta di un costume dinastico, ma per lo più il leader supremo appare come un ragazzone pingue con una buffa pettinatura. La sua pinguedine, inoltre, è considerata esteticamente negativa in occidente, dove predomina il modello della magrezza e della forma fisica, e dove i leader politici non sono da molto tempo modelli estetici a priori. 


\section{Ocullatiux_sagi}

Giampaolo Proni | Chiome a confronto | DOI: 10.12977/ocula2019-14

Questa paffutezza sorridente, associata a una pettinatura così gonfia in alto e con rasatura laterale, veicola l'immagine di un grande bambino cicciottello.

L'interpretazione occidentale non coglie inoltre i tratti mitici ed eroici dell'immagine dinastica kimiana, poiché ignora il codice entro il quale essa si è formata.

In un certo senso assistiamo all'inverso del meccanismo estetico che fece dell'immagine di Ernesto Che Guevara un'icona degli anni 60-70. Guevara, in particolare nel ritratto più diffuso, non aveva probabilmente intenzione di imitare i giovani alternativi e hippy occidentali, ma senza volerlo realizzava in pieno i canoni estetici della cultura alternativa ${ }^{41}$.

7.4.4 Che cosa Kim vuole dire con la propria immagine?

Che cosa possiamo dire delle intenzioni comunicative di Kim? Innanzitutto, per quanto riguarda il suo paese, possiamo essere certi che i significati che intende veicolare denotativamente sono espressi in maniera chiara e sufficientemente marcata. Gli ordini, i suggerimenti (che sono ordini anch'essi), le simpatie e le antipatie, gli slogan e i discorsi del Leader Supremo sono comunicati in ogni modo e costantemente ribaditi. Il livello connotativo è ugualmente chiaro. Tutti i simboli del potere e della prosperità accompagnano costantemente Kim, e la presenza dei suoi avi, che lo proteggono e lo legittimano, è continua, affidata, come abbiamo visto, agli onnipresenti ritratti ufficiali.

Che cosa Kim intenda dire all'Occidente ${ }^{42}$ è meno chiaro, e il dubbio che non senta l'esigenza di esprimere particolari contenuti è legittimo. Kim raramente rilascia interviste ${ }^{43}$, ma pronuncia discorsi e dichiarazioni ufficiali. A parte i contenuti politici e gli scambi di minacce con Trump, seguite da profferte di amicizia e poi da una nuova rottura, l'immagine di Kim non pare rivol-

${ }^{41}$ Per la storia del suo più famoso scatto vedi Wikipedia Guerrillero Heroico.

42 È sempre difficile trovare un termine per definire la cultura originata e condivisa in Europa, USA e Canada e, parzialmente in Russia, Giappone e America Latina, nonché estesa al resto del mondo nel corso del colonialismo europeo e dell'espansione economico-mediatica USA. Il termine 'occidentale' riferito alle aree geografiche e culturali si può considerare in un certo senso analogo al 'vestire all'occidentale', stile diffuso in tutto il mondo ma assimilato a titolo diverso in un paese e nell'altro. In Giappone è uno dei costumi usati; in India distingue anche uno strato sociale; in altri paesi riguarda le persone di una certa età; in Europa è il modo standard di abbigliarsi, ecc. Grandi differenze dipendono anche dai canali socio-mediatici sui quali sono sintonizzati i diversi gruppi sociali. In molti paesi emergenti solo la popolazione anglofona è coinvolta nelle reti dell'informazione globale; in Europa gran parte della popolazione è esposta ai media nelle lingue nazionali. In sintesi, 'occidentale' è un'area culturale simile a quella delle teorie semantiche prototipiche, che in linea generale producono una mappa policentrica (ma non priva di gerarchie) della diffusione dei valori culturali. Le ipotesi di comprensione dei messaggi da parte delle diverse componenti culturali del mondo dipendono dalla conoscenza dei filtri interpretativi che ognuna di esse applica, insieme alla consapevolezza di quelli della cultura dell'osservatore, i più difficili da rilevare. La semiotica ha strumenti adeguati a rappresentare queste relazioni, ma l'efficacia della loro applicazione non dipende solo da aspetti formali. Questo articolo si limita a conclusioni fondate su unità culturali ben rilevabili sui media.

43 Un raro caso è documentato in $\mathrm{BBC}$ (2019a). 
Giampaolo Proni | Chiome a confronto | DOI: 10.12977/ocula2019-14

ta al pubblico occidentale ${ }^{44}$. L'abito a giacca indossato durante il discorso di capodanno pare indirizzato prevalentemente ai coreani. In ogni caso, in generale si attiene al suo costume, e dunque usa il proprio linguaggio senza curarsi della possibile traduzione. L'immagine è costruita secondo un codice ristretto ai leader comunisti asiatici, e forse può essere compreso da chi in occidente ha maggiore familiarità con queste figure e queste ideologie.

In generale, tuttavia, quest'aspetto esteriore si impone all'immaginario occidentale, rendendo difficile far collimare l'interpretante di un allegro bambino paffuto proveniente da un grande parco tematico sul comunismo con la sua fama di dittatore sanguinario e affamatore del suo popolo ${ }^{45}$. Kim e il suo regime appaiono ai nostri occhi estranei al presente, alieni, abitanti di un set cinematografico. Sotto questo aspetto l'intenzione di Kim e del suo apparato è sicuramente scarsamente compresa in Occidente, e se vi era una strategia specifica di trasferire i valori locali a livello globale, non ha avuto alcun successo.

Tutta l'interazione tra Trump e Kim è pervasa così da un tono di commedia, di operetta, sia nella fase delle minacce e degli insulti reciproci sia in quella della riconciliazione e dell'amicizia. Il dialogo tra i due leader non riesce a cancellare l'impressione di una manifestazione di amicizia che pare quella tra due bambini capricciosi, entrambi abituati ad essere al centro dell'attenzione, del tutto personale e indifferente all'immensa platea che li osserva. Anche l'imprevisto fallimento dei colloqui di Hanoi ha rafforzato questa impressione ${ }^{46}$.

Nel complesso, per il pubblico occidentale l'immagine di questa relazione è piuttosto vaga e sostanzialmente solo spettacolare e l'immagine di Trump e della presidenza USA non ne escono sicuramente rafforzate. Quale sia l'effetto in Corea del Nord è difficile dirlo, ma probabilmente i media statali controllano ogni messaggio e forniscono versioni adatte a difendere e esaltare le posizioni patriottiche.

\section{Terzo livello: il significato pragmatico}

\subsection{Conseguenze ipotizzabili della pettinatura di Trump}

Quali conseguenze pratiche deriverebbero dalla nostra ipotesi sull'autismo nella presentazione di sé di Trump?

La prima è che la comprensibilità della sua immagine sarà inferiore a quella di una comunicazione adeguata al livello del ricevente, e una bassa com-

44 Di fatto esiste un apparato di comunicazione nord coreano che si avvale anche di social network, con traduzioni nelle lingue occidentali. Si tratta di un sistema, tuttavia, ancora simile ai tradizionali strumenti di propaganda dei regimi comunisti, che si occupa sostanzialmente di esaltare personalità e azioni della leadership.

45 Kim è quasi certamente il responsabile di una serie di esecuzioni di personaggi di alto livello tra i quali secondo alcuni commentatori il fratello. Vedi Boyle (2016), Wikipedia Hyon Yong-chol, Holloway (2018), Pleasance (2015), Wikipedia Kim Jongnam.

46 Mentre lavoravo a questo articolo un altro incontro tra Kim e Trump ha avuto luogo, nella zona demilitarizzata tra le due Coree (DMZ), il 30/6/2019, con breve preavviso. I risultati concreti, anche secondo osservatori internazionali, sono ancora dubbiosi. Vedi: <https://tinyurl.com/smufknc>, (online il 13/9/2019). 


\section{Ocullatiux_sagi}

Giampaolo Proni | Chiome a confronto | DOI: 10.12977/ocula2019-14

prensibilità pregiudica qualsiasi messaggio si intenda veicolare. La seconda è una valutazione della spontaneità dell'emittente maggiore di quanto sia veramente.

In un ambiente politico questo atteggiamento ha vantaggi e svantaggi. Un vantaggio è l'autonomia di opinione ed espressione. Un soggetto così 'distaccato' può apportare cambiamenti originali nel suo stile di comunicazione e di management. Il punto critico è che questi cambiamenti non sono del tutto consapevoli, perché, in un certo senso, sono narcisistici, presi senza considerare i punti di vista degli altri. Uno svantaggio è anche che, in una trattativa alla pari, il soggetto può non essere capace di farsi comprendere dall'interlocutore, senza neppure rendersene conto, e può sembrare un po' fuori tema, come se gli sfuggisse una parte del frame o conoscenza di sfondo richiesta dalla situazione. Questo non determina necessariamente un'inferiorità tattica o strategica. In politica emettere messaggi fuzzy, vaghi e/o contraddittori può dare un vantaggio, in quanto l'avversario è spiazzato nel negoziato. In sport come il basket, il rugby e il calcio, le finte sono parte essenziale del gioco: il portatore di palla accenna una mossa ma poi rapidamente ne fa un'altra. I giocatori inesperti a volte confondono le finte con l'indecisione. La differenza è che in una buona finta il giocatore sa che cosa intende fare, e così riesce a ingannare l'avversario ma non sé stesso. Nell'indecisione il giocatore può ingannare l'avversario o no, ma inganna sempre sé stesso, perché non sa che cosa vuol fare. A volte funziona, ma più spesso danneggia il giocatore, che perde il controllo dell'azione.

Le implicazioni dell'atteggiamento comunicativo di Trump, così come le abbiamo inferite dalla sua acconciatura usando gli strumenti semiotici, ci portano dunque a concludere che esso può portare alcuni vantaggi politici, ma anche svantaggi e, cosa peggiore di tutte, porta a una perdita di controllo su temi critici.

Il futuro ci dirà se queste previsioni saranno verificate ${ }^{47}$.

47 L'analisi è stata condotta tra l'estate e l'autunno del 2017. Fatti successivi che surrogano l'ipotesi ve ne sono stati. A inizio 2019, tra gli altri, possiamo sottolineare la decisione di ritirare le truppe americane dall'Afghanistan, criticata anche da alti membri dell'amministrazione, proprio per le incerte conseguenze, e la guerra commerciale con la Cina. Trump ha definito questo conflitto 'facile da vincere' (Trump 2018), ma le conseguenze effettive non sembrano rientrare del tutto nelle sue intenzioni (vedi come esempio Mayeda and Baschuk 2018). Anche la più recente decisione di appoggiare apertamente il candidato alla presidenza del Venezuela Juan Guaidò (vedi Reuters 2019) al momento non sembra produrre conseguenze controllabili. Recentissimo è lo sviluppo del conflitto USA-Iran dopo l'attacco agli impianti petroliferi sauditi Aramco del settembre 2019, anch'esso in costante altalena tra previsioni di incontri bilaterali, rotture e minacce di azioni militari ed economiche, riavvicinamenti. In generale, la politica estera di una superpotenza privilegia, in situazioni non di emergenza, scelte di esito sicuro. Vi sono però anche fatti che portano a falsificare l'ipotesi. In particolare, si può obiettare che Trump non pare usare molto le finte, bensì agire con uscite anche troppo nette, fino ad essere rozze, ma non appare mai indeciso, semmai troppo deciso. Tuttavia, il vorticoso turnover di collaboratori e di alti funzionari di nomina presidenziale non si può attribuire solo alla loro incapacità di applicare una linea politica chiara e definita. 


\section{Deula $a^{\text {Plux_saggi }}$ \\ Occhio semiotico sui media | Semiotic eye on media}

Giampaolo Proni | Chiome a confronto | DOI: 10.12977/ocula2019-14

\subsection{Conseguenze ipotizzabili della pettinatura di Kim e conclusione}

Il confronto appare uno sketch, una sceneggiata dalla trama scontata e poco originale, recitata da due personaggi che si reggono entrambi su narrazioni finzionali, quella di Trump estetica e un po' autistica, quella di Kim isolata, provinciale e infantile. E questa marca di infantilismo non si riesce a cancellare dai loro look. Risulta difficile pensare che il dialogo dei nostri personaggi possa sollevarsi da un livello di superficiale messa in scena, sia nel contrasto sia nell'affratellamento: due estetiche che non possono incontrarsi, due sistemi che si manifestano con gli aspetti più impermeabili alla reciproca interazione. Un discorso che sembra sempre più mirare a un obiettivo puramente fatico. Tuttavia, se il discorso fatico è importante come base per l'interazione sociale laddove esiste una situazione sociale condivisa, è difficile definirlo come l'obiettivo fondamentale di un summit internazionale. Non può esistere una vera phatic communion tra capi di stato così lontani per cultura, linguaggio, biografia, che interagiscono in situazioni rigidamente controllate, mediante interpreti, condizionati da apparati che lavorano prima e dopo ogni incontro. Non si tratta di un incontro tra culture, solo di vertici dei leader.

Resta forte l'impressione che a Trump e a Kim interessi solo mostrare di dialogare, ma senza avere nulla da dire, e ancora meno da fare. La diplomazia non ignora certo questa strategia di contatto esibito, ma non fine a se stessa. La politica internazionale è in un certo senso all'opposto della phatic communion, soprattutto se orientata al sovranismo (come pare sia la posizione di Trump): ogni mossa dovrebbe portare vantaggi per il proprio paese. E diversi osservatori cominciano a valutare che chi sta ottenendo di più da questo altalenante confronto sia proprio Kim Jong-un, se non altro perché lo status quo lo favorirebbe.

Tornando ai due look, essi sono molto diversi ma entrambi orientati verso l'interno. Quello del presidente americano verso un compiacimento idiolettico individuale, solipsistico, quello di Kim verso la conferma di un ruolo e di una posizione impossibile da migliorare, di piena soddisfazione entro il sistema culturale di appartenenza, senza necessità di apertura. Tutt'al più, qualche escursione edonistica nella cultura popolare occidentale.

L'analisi semiotica ci restituisce insomma il confronto di due esteriorità estranee e separate ma che condividono il piacere egocentrico di vivere ciascuno nella propria eco chamber ${ }^{48}$. Tra le due, la più ampia pare quella di Kim Jong-un.

Se estendiamo il confronto al livello transculturale, cioè alla relazione semiotica tra la cultura nord coreana e quella occidentale/globale, abbiamo un buon esempio di relazioni internazionali 'across the wall', cioè una visione che vede il dialogo tra culture come un 'parlarsi attraverso un muro', un rapporto che rimarca la separazione oggettiva come condizione per una negoziazione.

Questo approccio privilegia la messa in scena del dialogo rispetto al fine del dialogo stesso, poiché la separazione tra i partecipanti è in ogni caso implicita nell'interazione. L'esibizione di muri e il ribadire differenze e distinzioni è una

\footnotetext{
48 Vedi Wikipedia Echo chamber.
} 


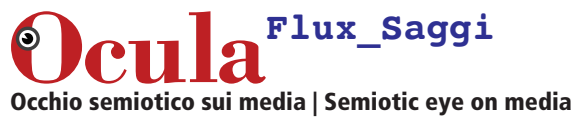

Giampaolo Proni | Chiome a confronto | DOI: 10.12977/ocula2019-14

tattica dilatoria. Nelle negoziazioni la si adotta quando si ritiene di avere il tempo a favore o si attendono eventi vantaggiosi. Ovviamente comporta un rischio e allontana la conclusione. In termini di diplomazia, i due sistemi si ritrovano a oscillare tra la difesa delle proprie posizioni e i vantaggi che il dialogo può produrre, senza giungere a una nuova stabilità. In questo caso, se si arriva a una dilazione (o rottura temporanea) per decisione di una parte, è evidente che questa ritiene di non esserne penalizzata. Tuttavia, la parte 'lasciata' non ha altra scelta diplomatica se non continuare a esercitare la pressione che ha portato a valutare necessario il confronto. Se una delle due parti potesse prevalere senza trattare, l'avrebbe fatto. Si ha ancora l'idea, tuttavia, che le due parti non siano in grado di trovarsi su una visione comune della necessità di trattare ma si siano limitate a mettere sul tavolo le reciproche richieste e disponibilità ${ }^{49}$.

Proprio qui si rivela la debolezza della visione 'divisiva' delle relazioni interculturali: le due parti non riescono a costruire una descrizione comune, non sono capaci di condividere una narrazione forse per il timore di perdere la propria identità. Entrambe o almeno una di esse. In tali condizioni, si genera un feed back negativo che mette in dubbio la ragione stessa del dialogo.

Vediamo il limite logico delle ideologie sovraniste: qualsiasi tipo di interazione comporta una visione condivisa, qualunque essa sia. Essa non coinvolge l'identità, a meno che essa non sia vista in maniera superficiale, costituita da discorsi temporanei e non da sistemi profondi di valori.



Graffiti dell'artista australiano Lush Sux sui pilastri settentrionali del Ponte Schweden a Vienna: il presidente USA Donald Trump e il capo di stato nordcoreano Kim Jong-un con le acconciature invertite. (Foto: (C) Bwag/Wikimedia).

49 In questo parziale modello non ho considerato la complessità dei sistemi politici che stanno alle spalle dei due leader. È evidente che il leader possiede una visione e una capacità decisionale individuale, ma essa è comunque influenzata (molto o poco e in modi diversissimi) da molteplici soggetti individuali e collettivi. Dal punto di vista geopolitico la Corea del Nord rappresenta una pedina di grande importanza nello scacchiere asiatico. Non è un caso se Trump ha ventilato, tra i vantaggi di un possibile accordo, l'insediamento massiccio di industrie in Corea del Nord. Così come la Germania, una Corea unita potrebbe diventare un gigante economico. La Cina non può restare indifferente agli eventi, ma qui estenderei troppo le mie pretese competenze. 


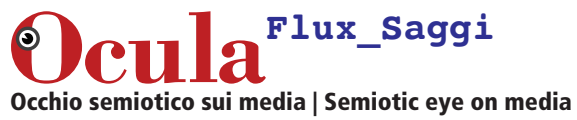

Giampaolo Proni | Chiome a confronto | DOI: 10.12977/ocula2019-14

\section{Riferimenti}

\section{Testi campione}

\section{Donald Trump}

TC-o1 s.d. un popolare sito sulla pettinatura di Trump: <http://trumpdonald.org/>.

TC-02 2015a, "A photo history of Trump's hairdo", in Vanity Fair, September 2015, $<$ https://tinyurl.com/y3nfaadv>, (8/8/2017).

TC-O3 2015b, "Prince Charles, Prince of Wales, is presented an Australian Team shirt by Australian Team Manager Gavin Dovey at SWALEC Stadium on July 6, 2015 in Cardiff, United Kingdom”, in Zimbio, July 5, 2015, <https://tinyurl.com/t3gvx25>, (8/8/2017).

TC-04 2016, "Donald Trump Lets Jimmy Fallon Mess Up His Hair”, in You Tube, September 15, 2016, <https://tinyurl.com/sjj2zu2>, (11/11/19).

TC-05 Mitgang, C. (2015), "A hairdresser explains why Donald Trump's hair looks like that”, in Quartz, December 18, 2015, $<$ https://tinyurl.com/wgebtqt>, (8/8/2017).

TC-o6 Page, M. (2014), "Donald Trump Hair Mystery-Combover, Toupee, Transplant, Follicle Faux Pas?”, in Popdust, September 19, 2014, <https://tinyurl.com/wzqr8ng>, (8/8/2017).

TC-07 Samson, P. (2016), "The truth about Donald Trump's hair: Former hairdresser reveals the lacquer, home cuts... and if it's real”, in Mirror, November 11, 2016, <https://tinyurl.com/jnqpedr>, (8/8/2017).

TC-o8 Mail Online (2014), "Watch the hair! Donald Trump accepts the Ice Bucket Challenge”, in Daily Mail, 2014, $<$ https://tinyurl.com/qnq4na6>, (8/8/2017).

TC-o9 Woolf, J. (2016), “Trump’s Tie Is Held Together With Scotch Tape”, in Huffington Post, December 2, 2016, <https://tinyurl.com/j3xby6v>, (8/8/2017).

\section{Kim Jong-Un}

TC-10 Ashcraft, Brian (2015), "Kim Jong-un: A Haircut Odyssey”, Kotaku, 20/2/2015, <https://tinyurl.com/sy23fy4>, (12/4/2019).

TC-11 BBC (2018), “Trump receives 'warm' letter from Kim about new summit”, $11 / 9 / 2018$, <https://tinyurl.com/u5aekkj>, (6/5/2019).

TC-12 Ferrier, Morvenna (2015), "Kim Jong-un defies gravity with new haircut", The Guardian, 20/2/2015, <https://tinyurl.com/sc8muoq>, (12/4/2019).

TC-13 Tidey, A. (2019), "Trump and Kim to meet for second summit in February" Euronews, 19/1/2019, <https://tinyurl.com/ycwp6976>, (6/5/2019). 


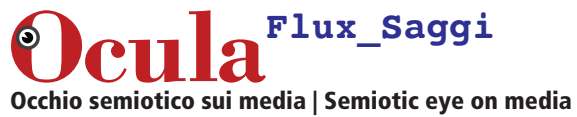

Giampaolo Proni | Chiome a confronto | DOI: 10.12977/ocula2019-14

\section{Kim Il-Sung}

TC-14 Wikipedia (2019), Kim Il-sung, <https://tinyurl.com/vw6czyz>, (17/9/2019).

TC-15 Wikimedia (2019), Category: Portraits of Kim Il-sung, <https://tinyurl.com/wfwp8kz>, (17/9/2019).

\section{Kim Jong-Il}

TC-16 Wikipedia (2019), Category: Portraits of Kim Jong-il, <https://tinyurl.com/t3l2pzk>, (17/9/2019).

\section{Bibliografia}

Barthes, R. (1957), Mythologies, Editions du Seuil, Paris.

Barthes, R. (2006), Il senso della moda. Forme e significati dell'abbigliamento, a cura di G. Marrone, Einaudi, Torino ("Histoire et sociologie du vêtement", Annales, luglio-settembre 1957; "Langage et vêtement”, Critique, marzo 1959).

Baudelaire, C. (2004), Il pittore della vita moderna, Abscondita, Milano (1885, Le Peintre de la vie moderne).

BBC (2005), "N Korea wages war on long hair" (8/1/2005), <https://tinyurl.com/443ty>, (12/4/2019).

BBC (2015), “Alexis Tsipras: Leader who risked Greece’s euro future” (21/9/2015), <https://tinyurl.com/yxxfsrfx>, (4/5/2019).

BBC (2018), "North Korea’s Kim Jong-un gets 'first official portrait” (6/11/2018), $<$ https://tinyurl.com/ycxh4cth>, (4/5/2019).

BBC (2019a) "When Kim responded to a foreign reporter" (video), <https://tinyurl.com/u307kr7>, (4/5/2019).

BBC (2019b) “Kim Jong-un leaves North Korea for Vietnam by train” (24/2/2019), <https://tinyurl.com/yyy6vdjq>, (4/5/2019).

Berger, P.L. and Luckmann, T. (1966), The Social Construction of Reality. A Treaty in the Sociology of Knowledge, Penguin, London-New York.

Bieber, Nicholas (2017), "Kim back and sides: The bizarre 15 state-approved haircuts on offer in North Korea”, in Daily Star, 30/4/2017, <https://tinyurl.com/quowz4g>, (12/4/2019).

Boyle, D. (2016), "Kim Jong-un uses an anti-aircraft gun to execute one high-ranking official for SLEEPING in a meeting and another for coming up with his own idea", Mailonline, 30 August 2016, <https://tinyurl.com/wbkryyq>.

Christian, S. (2017), Donald Trump's Tie Taping Has Officially Gone Too Far, in "Esquire", March 7, 2017, <https://tinyurl.com/jukoppw>. 


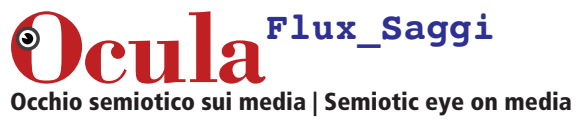

Giampaolo Proni | Chiome a confronto | DOI: 10.12977/ocula2019-14

Collins, R. (2012), Marked for Life: SONGBUN, North Korea's Social Classification System, The Committee for Human Rights in North Korea, Washington (https://www.hrnk.org/).

Cool Men’s Hair (s.d.) “John F. Kennedy’s Hairstyle: Get The Best Iconic Locks”, $<$ https://tinyurl.com/sn4kqxh>, (4/5/2019).

Corriere.it 1/4/2018 "Kim Jong-un tra le star del K-pop. È il primo concerto di artisti sudcoreani a Pyongyang da oltre 10 anni", <https://tinyurl.com/t3ofbr9>, (11/11/19).

Di Donato, T. E. (2015), 5 Research-Backed Reasons We Wear Makeup, in "Psychology Today", February 6, 2015, <https://tinyurl.com/jlxv2xq>, (9/8/2017).

Di Stefano, E. (2012), Iperestetica. Arte, natura, vita quotidiana e nuove tecnologie, in "Aesthetica Preprint", August 2012, <https://tinyurl.com/wm8gr76>.

Eco, U., and Sebeok, Th. A. (1983), The Sign of Three, Indiana University Press, Bloomington.

Eco, U. (1975), Trattato di semiotica generale, Bompiani, Milano.

Eco, U. (1979), The Role of the Reader. Explorations in the Semiotics of Texts, Indiana University Press, Bloomington.

Ellis, B.E. (1991), American Psycho, Vintage Books, New York.

Flügel, J.C. (1930), Psychology of Clothes, Hogarth, London.

Fuchs, C. (2017), Langue \& parole, linguistique, in "Encyclopædia Universalis”, April 2017 <https://tinyurl.com/v7wqhdo>.

Goffman, E. (1956), The Presentation of Self in Everyday Life, University of Edinburgh Social Sciences Research Centre, Edinburgh.

Goffman, E. (1963), Behavior in Public Places. Notes on the Social Organization of Gatherings, The Free Press, New York.

Grappe, M. (2018), Corea del Nord: la metamorfosi (video 13') Arte TV, disponibile dal 25/02/2019 fino al 23/06/2021., <https://tinyurl.com/wb8z638>,(4/5/2019).

Greimas. A.J. e Courtés, J. (1979), Semiotica. Dizionario ragionato della teoria del linguaggio, , a cura di Paolo Fabbri, Milano, Bruno Mondadori, 2007 (1979, Sémiotique: dictionnaire raisonné de la théorie du langage, Hachette, Paris).

Holloway, H. (2018), "Caught NAPPING: North Korean general risks EXECUTION after dozing off during Kim's speech”, DailyStar, 22 April 2018, $<$ https://tinyurl.com/wz7egr4>.

Jakobson, R. (1960), Closing Statements: Linguistic and Poetics, in T. A. Sebeok (ed.), Style in Language, The M.I.T. Press, New York-London, pp. 350-379.

Jones, T. (2013), Well she does not qualify for a bus pass..., in "Daily Mail”, May 23, 2013,

<https://tinyurl.com/oetb26a>. 


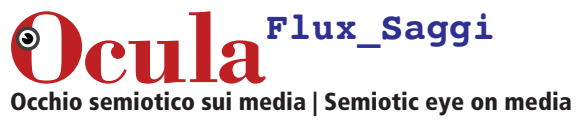

Giampaolo Proni | Chiome a confronto | DOI: 10.12977/ocula2019-14

Kawamura, Y. (2005), Fashion-ology. An Introduction to Fashion Studies, Berg, Oxford-New York.

Kawamura, Y. (2011), Doing Research in Fashion and Dress. An Introduction to Qualitative Methods, Bloomsbury, New York.

Knight, C. (2008), Honest Fakes' and Language Origins, in "Journal of Consciousness Studies”, 15, nn. 10-11, pp. 236-248.

König, R. (1973), The Restless Image: A Sociology of Fashion, transl. by E. Bradley. George Allen \& Unwin, Ltd. , London.

Little, B. (2018), "Kim Jong-il Was So Obsessed With Film He Kidnapped an Actress", History Channel,

<https://tinyurl.com/u2bja59>, (30/4/2019).

Loomes, P. (2019), "Kim Jong-un tries new look as he warns US is leaving North Korea with 'no option' but to change tack" News.com.au, January 2, 2019, <https://tinyurl.com/ybgnnzw6>, (30/4/2019).

Lotman Jurij M. e Uspenskij, Boris A. (1975), Tipologia della cultura, Bompiani, Milano, 1975.

Malinowski, B. (1923), The Problem of Meaning in Primitive Languages, in C.K. Ogden and I.A. Richards, The Meaning of Meaning, Harcourt, Brace \& World, New York 1989, pp. 296-336.

Matteucci, G. (2016), The Aesthetics as a Matter of Practices: Form of Life in Everydayness and Art, in "Comprendre", v. 18, n. 2, 2016, pp. 9-28.

Mayeda, A. and Baschuk, B. (2018), "Can Trump Win an Escalating Trade War Against China?: QickTake”, bloomberg.com 18/9/2019, <https://tinyurl.com/w5l322l>, (11/11/19).

McCracken, G. (1985), Clothing as a language: an object lesson in the study of the expressive properties of material culture, in B. Reynolds, M. Stotts (eds.), Material Anthropology, University Press of America, New York, pp. 110-122.

McCracken, G. (1995), Big Hair: A Journey into the Transformation of Self, Viking Press, Toronto.

McMurtrie, R. J. (2010), Bobbing for power: an exploration into the modality of hair, in "Visual Communication", v. 9, n. 4, 2010, pp. 399-424.

Muzzarelli, F., (2015), "Dandy e Neodandy. Fashion Visual Icons da La dolce vita a La grande bellezza”, Bianco e Nero, LXXVI, 581, gennaio-aprile 2015, pp. 27-35.

Ossola, A. (2018), "Kim Jong Un Always Wears the Same Suit - This Is What It Means" Racked, 12/6/2018, <https://tinyurl.com/yc75yweq>, (4/5/2019).

Padden, Brian (2014), "No Sign of Kim Jong Un on Important Anniversary", Voanews.com, October 10, 2014, <https://tinyurl.com/vyzcfzn>, (11/11/19).

Pasolini, P.P. (1973), “Contro i capelli lunghi”, Corriere della Sera, 7/1/1973.

Peirce, C. S. (1878), How To Make Our Ideas Clear, in 1982-in progress WR 3.257276. 


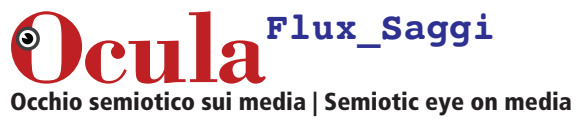

Giampaolo Proni | Chiome a confronto | DOI: 10.12977/ocula2019-14

Peirce, C. S. (1887-1888), A Guess at the Riddle in 1982-in progress, WR 6.165-210, item 22.

Peirce, C. S. (c.1907), A Survey of Pragmaticism, in 1931-58 CP 5.11-13; 5.464-496.

Peirce, C. S. (1931-1958), Collected Papers of Charles Sanders Peirce. 8 voll. Voll. I-VI, 1931-1935, C. Hartshorne, P. Weiss, eds. Voll. VII-VIII, 1958, A. Burks (ed.), Harvard University Press, Cambridge, MA.

Peirce, C. S. (1982-in progress) Writings of Charles Sanders Peirce. A Chronological Edition, Indiana University Press, Bloomington.

Pleasance, C. (2015), "North Korean despot Kim Jong-Un has executed 70 officials ...” MailOnline, 9 July 2015 ,

$<$ https://tinyurl.com/vyadsle>.

Proni, G. (2001), Il riporto e la rasatura: come si formano metafore socio-percettive, in "Ocula", n. 1, March 2001, <https://tinyurl.com/wx8c88z>, (8/8/2017).

Proni, G. (2002), Outlines for a semiotic analysis of objects, in "Versus", nn. 91-92, January-August 2002, pp. 37-59.

Proni, G. (2007), Fast Fashion, Fast Shopping: a semiotic approach to practices of consumption of young consumers", in "Ocula" n. 8, July 2007, <https://tinyurl.com/uxk3nwa>, (10/8/2017).

Proni, G. (2010), Per una semiotica del gossip, in F. Muzzarelli (ed.), Gossip. Moda e modi del voyerismo contemporaneo, Bologna University Press, Bologna, pp. 113-119.

Proni, G. (2015), Linguaggio e vestito: Roland Barthes e Charles Peirce, in "Rivista Italiana di Filosofia del Linguaggio", 2015,

$<$ https://tinyurl.com/qkd4hpd>.

Reuters (2019), “Trump reaffirms U.S. support in call with Venezuela's Guaido”, Reuters.com 30/1/2019, <https://tinyurl.com/ukega7l>, (5/5/2019).

Siddiqui, D. "Portraits of a dynasty: North Korea's ever-present Kims", Reuters, World News, September 25, 2018, <https://tinyurl.com/tr2ewjr>, (30/4/2019).

Simmel, G. (1957), Fashion, in "The American Journal of Sociology", v. 62, n. 6, May 1957, pp. 541-558.

Spencer (1898), The Principles of Sociology, 3 vol.s, D. Appleton and Company, New York.

Tarde, G. (1890), Les lois de l'imitation, Felix Alcan, Paris.

Traini, S. (2005), "L’eredità di Lotman”, E/C n. 2 del 17 gennaio 2005.

Traini, S. (2013), Le basi della semiotica, Bompiani, Milano 2013.

Trump, D.J. (2018), Twitter @realDonaldTrump 2/3/2019 02:50, $<$ https://tinyurl.com/su5n4sx>, (5/5/2019).

Wikipedia (2019), Coda (acconciatura), <https://tinyurl.com/tlzagcj>, (16/9/2019). 


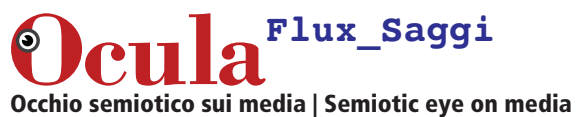

Giampaolo Proni | Chiome a confronto | DOI: 10.12977/ocula2019-14

Wikipedia (2019), Constitution of NorthKorea, $<$ https://tinyurl.com/rse8laa>

Wikipedia (2019), Cult of personality, $<$ https://tinyurl.com/ya2svjx5>, (4/5/2019).

Wikipedia (2019), Echo chamber (media), <https://tinyurl.com/pxpdddo>, (5/5/2019).

Wikipedia (2019), French (tunic), <https://tinyurl.com/spqj3f2>, (4/5/2019).

Wikipedia (2019), Guerrillero Heroico, $<$ https://tinyurl.com/kaxk3km>, (4/5/2019).

Wikipedia (2019), Hyon Yong-chol, , <https://tinyurl.com/uv2hyfm>, (11/11/19).

Wikipedia (2019), Kim Il-sung and Kim Jong-il portraits, <https://tinyurl.com/uho6acj>, (4/5/2019).

Wikipedia (2019), Kim Jong-nam, $<$ https://tinyurl.com/zlwglbu>, (4/5/2019).

Wikipedia (2019), Kim Jong-un, $<$ https://tinyurl.com/ppec3ku>, (11/11/19).

Wikipedia (2019), Mao suit, <https://tinyurl.com/qwvf86x>, (4/5/2019).

Wikipedia (2019), Mao Zedong, $<$ https://tinyurl.com/tmtvpqk>, (22/5/2019).

Wikipedia (2019), Mansudae Art Studio, $<$ https://tinyurl.com/umec2pd>, (22/5/2019).

Wikipedia (2019), North Korean cult of personality, <https://tinyurl.com/y97wghjj>, (4/5/2019).

Wikipedia (2019), North Korean leaders' trains, <https://tinyurl.com/s5xte82>, (4/5/2019).

Wikipedia (2019), Residences of North Korean leaders, <https://tinyurl.com/ww5vpn2>, (4/5/2019).

Wikipedia (2019), Ryongsong Residence, $<$ https://tinyurl.com/yaamk3f3>, (4/5/2019).

Wikipedia (2019), Songbun, <https://tinyurl.com/rmlc59m>, (16/9/2019).

Wikipedia (2019), Stalin tunic, $<$ https://tinyurl.com/sq6d67x>, (4/5/2019).

Wood, Vincent (2017), "Kim Jong-un FORBIDS North Koreans from COPYING his hair as state approved list revealed", in Express, 18/4/2017, $<$ https://tinyurl.com/r8r47cy>, (12/4/2019).

Wuyiran (2018) Right\&Relationship, 6/12/2018, $<$ https://tinyurl.com/r56hst6>, (contiene ideogrammi) (4/5/2019). 